\title{
Dynamic equations for a fully anisotropic piezoelectric rectangular plate
}

\author{
K. Mauritsson ${ }^{1}$, P.D. Folkow* \\ Department of Applied Mechanics, Chalmers University of Technology, SE-412 96 Göteborg, Sweden
}

\section{A R T I C L E I N F O}

\section{Article history:}

Received 18 December 2013

Accepted 18 February 2015

\section{Keywords:}

Piezoelectric plate

Power series

Asymptotic

Orthotropic

Eigenfrequency

Laminate

\begin{abstract}
A B S T R A C T
A hierarchy of dynamic plate equations based on the three dimensional piezoelectric theory is derived for a fully anisotropic piezoelectric rectangular plate. Using power series expansions results in sets of equations that may be truncated to arbitrary order, where each order set is hyperbolic, variationally consistent and asymptotically correct (to all studied orders). Numerical examples for eigenfrequencies and plots on mode shapes, electric potential and stress distributions curves are presented for orthotropic plate structures. The results illustrate that the present approach renders benchmark solutions provided higher order truncations are used, and act as engineering plate equations using low order truncation.
\end{abstract}

(c) 2015 Elsevier Ltd. All rights reserved.

\section{Introduction}

Piezoelectric materials have been used widely in applications for sensing and actuation purposes in recent years. As piezoelectric sensors and actuators usually are thin in comparison to relevant wavelengths, the analyzes of thin piezoelectric layers, such as beams, plates and shells, have attained considerable research interest. Many references to work on higher-order piezoelectric plate theories prior to 2000 are given in the review article by Wang and Yang [1]. Further references to laminated piezoelectric plates are presented in [2] while [3] presents a classification and comparison among higher-order piezoelectric plate models based on power series expansions. A more recent review article on three dimensional approaches for piezoelectric plates is presented by Wu et al. [4].

Plate theories for various material configurations were developed in the 50's by Mindlin, among which piezoelectric plates were addressed in [5]. This work was later generalized by Tiersten and Mindlin [6] and Mindlin [7] where two-dimensional equations for a piezoelectric plate were systematically derived using power series expansions for the mechanical and electric displacements. Bugdayci and Bogy [8] and Lee et al. [9,10] used trigonometric series expansions for piezoelectric plates, which provides an alternative approach for analyzing plate vibrations more suitable for high-

\footnotetext{
* Corresponding author. Tel.: +46 31 7721521; fax: +4631772 3827 .

E-mail addresses: karl.mauritsson@his.se (K. Mauritsson), peter.folkow@ chalmers.se (P.D. Folkow).

1 Present address: School of Technology and Society, University of Skövde, SE-541 28 Skövde, Sweden.
}

frequency modes. More recently developed plate theories using various sorts of series expansions of the displacements and the electric potential for both single and laminated piezoelectric plates can be found in [11-24]. These expansions are either using a few power series terms, or written on a general higher order fashion that may be used to render solutions that converge to the three dimensional solutions. Exact three dimensional analyses for single and laminated piezoelectric plates having mixed (simply supported) boundary conditions are treated in [25-28]. Numerical methods such as finite element analysis (FEA) has been adopted on both classical and higher order series expansion theories [29-34].

Recently Mauritsson et al. [35] have derived plate equations for a homogenous fully anisotropic elastic plate, using a systematic power series expansion approach, previously adopted for isotropic rods, beams, shells and plates [36-40]. The same general approach has been applied to various piezoelectric layer configurations [4143]. In the present paper the work in [35] is extended to cover anisotropic piezoelectric plates. The method aims at systematically develop a hierarchy of equations for general piezoelectric plates using power series expansions in the thickness coordinate of the displacement components and the electric potential. Insertion of these expansions into the three dimensional equations of motion leads to recursion relations among the expansion functions, which can be used to eliminate all but some of the lowest order expansion functions. Hereby all fields can be expressed in a finite number of expansions functions without performing any truncations. The power series expansions are subsequently inserted into the three dimensional boundary conditions at the upper and the lower surfaces of the plate. These boundary conditions represent a set of eight scalar equations of motion, including eight unknown 
expansion functions, which constitute the system of plate equations. Using variational calculus, the pertinent edge boundary conditions for rectangular plates are obtained in an equally systematic manner. This hierarchy of piezoelectric plate equations can be truncated to any order in the thickness where each studied truncation order is asymptotically correct in line with [35,37,38,44].

The present approach generally differs in several respects from the cited works using power series expansion on piezoelectric plates. The main issues concern the derivation of exact recursion relations where only the lowest order expansion terms need to be considered. Another object is the procedure when collecting terms for the truncation process, resulting in variationally consistent equation systems that are asymptotically correct. It should also be noticed that the present equations are not confined to the static case. Moreover, the plate configuration may be of arbitrary anisotropy without any symmetry classes. One advantage with such a general analysis is that all other cases can be obtained as special cases. The previously derived plate equations for the fully anisotropic elastic case [35] can also be obtained as a special case. As a fully anisotropic, piezoelectric material is described by 21 independent stiffness constants, 18 independent piezoelectric coupling constants and six independent dielectric constants, the explicit expressions for the coefficients in the plate equations become very complicated. For this reason the plate equations for the most general case are derived in a very compact form as four matrix equations, including matrix operators which are recursively defined using the commercial code Mathematica. ${ }^{2}$ Hereby it is straightforward to study all types of anisotropy configurations.

As the material configuration for a fully anisotropic material results in complicated expressions, it is natural to study simpler cases of orthotropic plates more in detail. Here the eight plate equations can be added and subtracted in pairs to obtain two uncoupled systems of equations, each of them including four equations and four unknowns. The two uncoupled systems correspond to the symmetric (in-plane) and antisymmetric (out-of-plane) part of the motion, respectively. These equations, including the edge boundary conditions, are explicitly given for the lower truncation orders. To validate the present plate equations results for dispersion curves, eigenfrequencies as well as potential, displacement and stress distribution curves are presented. Both single and laminated plates are studied and comparisons are made to other approximate theories as well as the exact three dimensional theory. The results illustrate both the benchmark property of the higher order truncations and the efficiency of the lower order engineering equations.

\section{Problem formulation}

Consider a homogeneous piezoelectric plate of thickness $2 \mathrm{~h}$ according to Fig. 1. The material is fully anisotropic with density $\rho$. The basic equations governing the motion in a piezoelectric continuum are written with abbreviated subscripts [45] as

$$
\begin{aligned}
& \nabla_{i J} T_{J}=\rho \partial_{t}^{2} u_{i}, \\
& \nabla_{i} D_{i}=0 .
\end{aligned}
$$

Here vector subscripts are expressed through lower case letters $i=x, y, z$, while abbreviated subscripts are expressed through upper case letters $I=1,2,3,4,5,6$. The mechanical stress, mechanical displacement and electric displacement column matrices are defined through

$$
\left[T_{I}\right]=\left(T_{x x} T_{y y} T_{z z} T_{y z} T_{x z} T_{x y}\right)^{T}, \quad\left[u_{i}\right]=\left(u_{x} u_{y} u_{z}\right)^{T}, \quad\left[D_{i}\right]=\left(D_{x} D_{y} D_{z}\right)^{T} .
$$

\footnotetext{
${ }^{2}$ Registered trademark of Wolfram Research Inc.
}

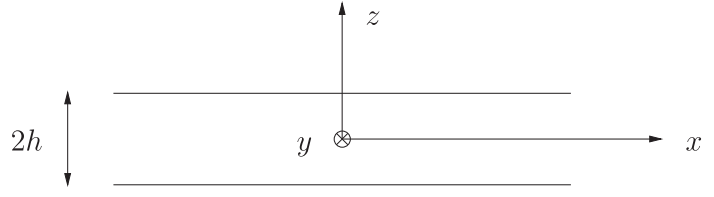

Fig. 1. The geometry.

The divergence vector $\nabla_{i}$ is defined in the usual way, while the divergence stress operator $\nabla_{i j}$ is represented in matrix form through

$\left[\nabla_{i j}\right]=\left(\begin{array}{cccccc}\partial_{x} & 0 & 0 & 0 & \partial_{z} & \partial_{y} \\ 0 & \partial_{y} & 0 & \partial_{z} & 0 & \partial_{x} \\ 0 & 0 & \partial_{z} & \partial_{y} & \partial_{x} & 0\end{array}\right), \quad\left[\nabla_{l j}\right]=\left[\nabla_{i j}\right]^{T}$.

Partial derivatives are expressed as $\partial_{x}=\partial / \partial_{x}$ and so on.

For a linear elastic, piezoelectric material the constitutive equations that express the mechanical stresses and the electric displacements in terms of the mechanical displacements and the electric potential, are

$T_{I}=c_{I J} \nabla_{J k} u_{k}+e_{I j} \nabla_{j} \Phi$

$D_{i}=e_{i j} \nabla_{J k} u_{k}-\epsilon_{i j} \nabla_{j} \Phi$.

Here the quasistatic approximation is applied, i.e. the electric field is given as the gradient of the electric potential

$E_{i}=-\nabla_{i} \Phi$.

The various material parameters appearing in (2.5) and (2.6) are the 21 independent stiffness constants collected in the symmetric $6 \times 6$ matrix

$\left[c_{I J}\right]=\left(\begin{array}{llllll}c_{11} & c_{12} & c_{13} & c_{14} & c_{15} & c_{16} \\ c_{12} & c_{22} & c_{23} & c_{24} & c_{25} & c_{26} \\ c_{13} & c_{23} & c_{33} & c_{34} & c_{35} & c_{36} \\ c_{14} & c_{24} & c_{34} & c_{44} & c_{45} & c_{46} \\ c_{15} & c_{25} & c_{35} & c_{45} & c_{55} & c_{56} \\ c_{16} & c_{26} & c_{36} & c_{46} & c_{56} & c_{66}\end{array}\right)$,

the 6 independent dielectric constants collected in the symmetric $3 \times 3$ matrix

$\left[\epsilon_{i j}\right]=\left(\begin{array}{ccc}\epsilon_{x x} & \epsilon_{x y} & \epsilon_{x z} \\ \epsilon_{x y} & \epsilon_{y y} & \epsilon_{y z} \\ \epsilon_{x z} & \epsilon_{y z} & \epsilon_{z z}\end{array}\right)$,

and the 18 independent piezoelectric coupling constants collected in the $3 \times 6$ piezoelectric coupling matrix

$\left[e_{i j}\right]=\left(\begin{array}{cccccc}e_{x 1} & e_{x 2} & e_{x 3} & e_{x 4} & e_{x 5} & e_{x 6} \\ e_{y 1} & e_{y 2} & e_{y 3} & e_{y 4} & e_{y 5} & e_{y 6} \\ e_{z 1} & e_{z 2} & e_{z 3} & e_{z 4} & e_{z 5} & e_{z 6}\end{array}\right), \quad\left[e_{I j}\right]=\left[e_{i j}\right]^{T}$.

Insertion of (2.5) and (2.6) into (2.1) and (2.2) gives the governing equations for the displacements and the electric potential

$\nabla_{i J} c_{J K} \nabla_{K l} u_{l}+\nabla_{i J} e_{j k} \nabla_{k} \Phi=\rho \partial_{t}^{2} u_{i}$

$\nabla_{i} e_{i J} \nabla_{J k} u_{k}-\nabla_{i} \epsilon_{i j} \nabla_{j} \Phi=0$.

\section{Power series expansions}

To derive plate equations, the displacement components and the electric potential are expanded in power series in the thickness coordinate

$u_{i}(x, y, z, t)=\sum_{n=0}^{\infty} z^{n} u_{i}^{(n)}(x, y, t)$ 
$\Phi(x, y, z, t)=\sum_{n=0}^{\infty} z^{n} \Phi^{(n)}(x, y, t)$

Insertion of the series expansions (3.1) and (3.2) into the governing equations of motion (2.11) and (2.12) gives power series expansion equations in the thickness coordinate $z$. By collecting equal powers of $z$, the solution of the equation system for each power yields the recursion formulas

$$
\begin{aligned}
u_{i}^{(n+2)}= & \frac{1}{(n+1)(n+2)}\left[A_{i j}^{(0)} u_{j}^{(n)}+(n+1) B_{i j}^{(0)} u_{j}^{(n+1)}\right. \\
& \left.+F_{i}^{(0)} \Phi^{(n)}+(n+1) G_{i}^{(0)} \Phi^{(n+1)}\right], \\
\Phi^{(n+2)}= & \frac{1}{(n+1)(n+2) \epsilon_{z z}}\left[a_{i}^{(0)} u_{i}^{(n)}+(n+1) b_{i}^{(0)} u_{i}^{(n+1)}\right. \\
& \left.+f^{(0)} \Phi^{(n)}+(n+1) g^{(0)} \Phi^{(n+1)}\right],
\end{aligned}
$$

where $n=0,1,2, \ldots$. The introduced operators are defined as

$A_{i m}^{(0)}=M_{i j}\left[\delta_{j m} \rho \partial_{t}^{2}-\bar{\nabla}_{j K} c_{K L} \bar{\nabla}_{L m}-\frac{1}{\epsilon_{z z}} e_{j} \bar{\nabla}_{k} e_{k L} \bar{\nabla}_{L m}\right]$,

$B_{i m}^{(0)}=-M_{i j}\left[L_{j K} c_{K L} \bar{\nabla}_{L m}+\bar{\nabla}_{j K} c_{K L} L_{L m}+\frac{e_{j}}{\epsilon_{z z}}\left(l_{k} e_{k L} \bar{\nabla}_{L m}+\bar{\nabla}_{k} e_{k L} L_{L m}\right)\right]$,

$F_{i}^{(0)}=M_{i j}\left[\frac{1}{\epsilon_{z z}} e_{j} \bar{\nabla}_{k} \epsilon_{k l} \bar{\nabla}_{l}-\bar{\nabla}_{j K} e_{K l} \bar{\nabla}_{l}\right]$,

$G_{i}^{(0)}=M_{i j}\left[\frac{e_{j}}{\epsilon_{z z}}\left(l_{k} \epsilon_{k l} \bar{\nabla}_{l}+\bar{\nabla}_{k} \epsilon_{k l} l_{k}\right)-L_{j K} e_{K l} \bar{\nabla}_{l}-\bar{\nabla}_{j K} e_{K l} l_{l}\right]$,

$a_{k}^{(0)}=e_{i} A_{i k}^{(0)}+\bar{\nabla}_{i} e_{i J} \bar{\nabla}_{J k}$,

$b_{k}^{(0)}=e_{i} B_{i k}^{(0)}+l_{i} e_{i J} \bar{\nabla}_{j k}+\bar{\nabla}_{i} e_{i j} L_{J k}$,

$f^{(0)}=e_{i} F_{i}^{(0)}-\bar{\nabla}_{i} \epsilon_{i j} \bar{\nabla}_{j}$

$g^{(0)}=e_{i} G_{i}^{(0)}-l_{i} \epsilon_{i j} \bar{\nabla}_{j}-\bar{\nabla}_{i} \epsilon_{i j} l_{j}$,

where $\delta_{i j}$ is the Kronecker delta. Here new material matrices are introduced as

$\left[e_{i}\right]=\left(e_{z 5} e_{z 4} e_{z 3}\right)$,

$\left[C_{i j}\right]=\left(\begin{array}{lll}c_{55}+e_{z 5}^{2} / \epsilon_{z z} & c_{45}+e_{z 4} e_{z 5} / \epsilon_{z z} & c_{35}+e_{z 3} e_{z 5} / \epsilon_{z z} \\ c_{45}+e_{z 4} e_{z 5} / \epsilon_{z z} & c_{44}+e_{z 4}^{2} / \epsilon_{z z} & c_{34}+e_{z 3} e_{z 4} / \epsilon_{z z} \\ c_{35}+e_{z 3} e_{z 5} / \epsilon_{z z} & c_{34}+e_{z 3} e_{z 4} / \epsilon_{z z} & c_{33}+e_{z 3}^{2} / \epsilon_{z z}\end{array}\right)$,

where $\left[M_{i j}\right]=\left[C_{i j}\right]^{-1}$. Hence, $\left[C_{i j}\right]$ can be interpreted as a matrix of stiffened elastic constants. Moreover the introduced ordering matrices are

$$
\left[l_{i}\right]=(001), \quad\left[L_{i j}\right]=\left(\begin{array}{cccccc}
0 & 0 & 0 & 0 & 1 & 0 \\
0 & 0 & 0 & 1 & 0 & 0 \\
0 & 0 & 1 & 0 & 0 & 0
\end{array}\right), \quad\left[L_{I j}\right]=\left[L_{i j}\right]^{T} .
$$

The operators with an overline $\left(\bar{\nabla}_{i}, \bar{\nabla}_{i j}\right.$ and $\left.\bar{\nabla}_{I j}\right)$ are differential operators in the plate plane obtained through

$\nabla_{i}=\bar{\nabla}_{i}+\partial_{z} l_{i}, \quad \nabla_{i j}=\bar{\nabla}_{i j}+\partial_{z} L_{i J}, \quad \nabla_{I j}=\bar{\nabla}_{I j}+\partial_{z} L_{I j}$.

Consequently $\bar{\nabla}_{i j}$ and $\bar{\nabla}_{l j}$ are obtained by putting all derivatives with respect to $z$ to zero in (2.4).

The recursion relations (3.3) and (3.4) can be used repeatedly to express all expansion functions in the lowest-order ones with an upper index $n=0,1$. The expansion functions can then be written as

$u_{i}^{(n)}=\frac{1}{n !}\left[A_{i j}^{(n-2)} u_{j}^{(0)}+B_{i j}^{(n-2)} u_{j}^{(1)}+F_{i}^{(n-2)} \Phi^{(0)}+G_{i}^{(n-2)} \Phi^{(1)}\right]$,

$\Phi^{(n)}=\frac{1}{(n !) \epsilon_{z z}}\left[a_{i}^{(n-2)} u_{i}^{(0)}+b_{i}^{(n-2)} u_{i}^{(1)}+f^{(n-2)} \Phi^{(0)}+g^{(n-2)} \Phi^{(1)}\right]$, where $n=0,1,2, \ldots$

Eqs. (3.9) and (3.10) include recursively defined differential operators, given by (3.5) and the following extensions

$$
\begin{aligned}
& A_{i k}^{(n)}=B_{i j}^{(0)} A_{j k}^{(n-1)}+A_{i j}^{(0)} A_{j k}^{(n-2)}+\frac{1}{\epsilon_{z z}}\left[G_{i}^{(0)} a_{k}^{(n-1)}+F_{i}^{(0)} a_{k}^{(n-2)}\right], \\
& B_{i k}^{(n)}=B_{i j}^{(0)} B_{j k}^{(n-1)}+A_{i j}^{(0)} B_{j k}^{(n-2)}+\frac{1}{\epsilon_{z z}}\left[G_{i}^{(0)} b_{k}^{(n-1)}+F_{i}^{(0)} b_{k}^{(n-2)}\right], \\
& F_{i}^{(n)}=B_{i j}^{(0)} F_{j}^{(n-1)}+A_{i j}^{(0)} F_{j}^{(n-2)}+\frac{1}{\epsilon_{z z}}\left[G_{i}^{(0)} f^{(n-1)}+F_{i}^{(0)} f^{(n-2)}\right], \\
& G_{i}^{(n)}=B_{i j}^{(0)} G_{j}^{(n-1)}+A_{i j}^{(0)} G_{j}^{(n-2)}+\frac{1}{\epsilon_{z z}}\left[G_{i}^{(0)} g^{(n-1)}+F_{i}^{(0)} g^{(n-2)}\right], \\
& a_{j}^{(n)}=b_{i}^{(0)} A_{i j}^{(n-1)}+a_{i}^{(0)} A_{i j}^{(n-2)}+\frac{1}{\epsilon_{z z}}\left[g^{(0)} a_{j}^{(n-1)}+f^{(0)} a_{j}^{(n-2)}\right], \\
& b_{j}^{(n)}=b_{i}^{(0)} B_{i j}^{(n-1)}+a_{i}^{(0)} B_{i j}^{(n-2)}+\frac{1}{\epsilon_{z z}}\left[g^{(0)} b_{j}^{(n-1)}+f^{(0)} b_{j}^{(n-2)}\right], \\
& f^{(n)}=b_{i}^{(0)} F_{i}^{(n-1)}+a_{i}^{(0)} F_{i}^{(n-2)}+\frac{1}{\epsilon_{z z}}\left[g^{(0)} f^{(n-1)}+f^{(0)} f^{(n-2)}\right], \\
& g^{(n)}=b_{i}^{(0)} G_{i}^{(n-1)}+a_{i}^{(0)} G_{i}^{(n-2)}+\frac{1}{\epsilon_{z z}}\left[g^{(0)} g^{(n-1)}+f^{(0)} g^{(n-2)}\right],
\end{aligned}
$$

for $n=0,1,2, \ldots$, using the base terms

$$
\begin{array}{llll}
A_{i j}^{(-2)}=\delta_{i j}, & A_{i j}^{(-1)}=0, & B_{i j}^{(-2)}=0, & B_{i j}^{(-1)}=\delta_{i j}, \\
F_{i}^{(-2)}=0, & F_{i}^{(-1)}=0, & G_{i}^{(-2)}=0, & G_{i}^{(-1)}=0, \\
a_{i}^{(-2)}=0, & a_{i}^{(-1)}=0, & b_{i}^{(-2)}=0, & b_{i}^{(-1)}=0, \\
f^{(-2)}=\epsilon_{z z}, & f^{(-1)}=0, & g^{(-2)}=0, & g^{(-1)}=\epsilon_{z z} .
\end{array}
$$

Note that the recursion formulas (3.9) and (3.10) involve no approximations since they stem from the definition of the series expansions (3.1) and (3.2) and are as such exact. These may be obtained by using variational calculus as in [38]. Moreover, no truncations of the displacement terms have so far been performed, which is of crucial importance for the present method.

Insertion of the power series expansions (3.1) and (3.2) into the constitutive equations for the stresses (2.5) and for the electric displacements (2.6) gives

$T_{I}=\sum_{n=0}^{\infty} z^{n} T_{I}^{(n)}$

$D_{i}=\sum_{n=0}^{\infty} z^{n} D_{i}^{(n)}$

where

$$
\begin{aligned}
T_{I}^{(n)}= & c_{I J}\left(\bar{\nabla}_{J k} u_{k}^{(n)}+(n+1) L_{J k} u_{k}^{(n+1)}\right) \\
& +e_{I j}\left(\bar{\nabla}_{j} \Phi^{(n)}+(n+1) l_{j} \Phi^{(n+1)}\right), \\
D_{i}^{(n)}= & e_{i J}\left(\bar{\nabla}_{J k} u_{k}^{(n)}+(n+1) L_{J k} u_{k}^{(n+1)}\right) \\
& -\epsilon_{i j}\left(\bar{\nabla}_{j} \Phi^{(n)}+(n+1) l_{j} \Phi^{(n+1)}\right) .
\end{aligned}
$$

By using the recursion formulas (3.9) and (3.10) these fields may finally be expressed in the lowest-order terms

$$
\begin{aligned}
T_{I}^{(n)}= & \frac{1}{n !}\left[\left[c_{I J}\left(\bar{\nabla}_{J k} A_{k l}^{(n-2)}+L_{J k} A_{k l}^{(n-1)}\right)+\frac{e_{I j}}{\epsilon_{z z}}\left(\bar{\nabla}_{j} a_{l}^{(n-2)}+l_{j} a_{l}^{(n-1)}\right)\right] u_{l}^{(0)}\right. \\
& +\left[c_{I J}\left(\bar{\nabla}_{J k} B_{k l}^{(n-2)}+L_{J k} B_{k l}^{(n-1)}\right)+\frac{e_{I j}}{\epsilon_{z z}}\left(\bar{\nabla}_{j} b_{l}^{(n-2)}+l_{j} b_{l}^{(n-1)}\right)\right] u_{l}^{(1)} \\
& +\left[c_{I J}\left(\bar{\nabla}_{J k} F_{k}^{(n-2)}+L_{J k} F_{k}^{(n-1)}\right)+\frac{e_{I j}}{\epsilon_{z z}}\left(\bar{\nabla}_{j} f^{(n-2)}+l_{j} f^{(n-1)}\right)\right] \Phi^{(0)} \\
& \left.+\left[c_{I J}\left(\bar{\nabla}_{J k} G_{k}^{(n-2)}+L_{J k} G_{k}^{(n-1)}\right)+\frac{e_{I j}}{\epsilon_{z z}}\left(\bar{\nabla}_{j} g^{(n-2)}+l_{j} g^{(n-1)}\right)\right] \Phi^{(1)}\right],
\end{aligned}
$$




$$
\begin{aligned}
D_{i}^{(n)}= & \frac{1}{n !}\left[\left[e_{i J}\left(\bar{\nabla}_{J k} A_{k l}^{(n-2)}+L_{J k} A_{k l}^{(n-1)}\right)-\frac{\epsilon_{i j}}{\epsilon_{z z}}\left(\bar{\nabla}_{j} a_{l}^{(n-2)}+l_{j} a_{l}^{(n-1)}\right)\right] u_{l}^{(0)}\right. \\
& +\left[e_{i J}\left(\bar{\nabla}_{J k} B_{k l}^{(n-2)}+L_{J k} B_{k l}^{(n-1)}\right)-\frac{\epsilon_{i j}}{\epsilon_{z z}}\left(\bar{\nabla}_{j} b_{l}^{(n-2)}+l_{j} b_{l}^{(n-1)}\right)\right] u_{l}^{(1)} \\
& +\left[e_{i J}\left(\bar{\nabla}_{J k} F_{k}^{(n-2)}+L_{J k} F_{k}^{(n-1)}\right)-\frac{\epsilon_{i j}}{\epsilon_{z z}}\left(\bar{\nabla}_{j} f^{(n-2)}+l_{j} f^{(n-1)}\right)\right] \Phi^{(0)} \\
& \left.+\left[e_{i J}\left(\bar{\nabla}_{J k} G_{k}^{(n-2)}+L_{J k} G_{k}^{(n-1)}\right)-\frac{\epsilon_{i j}}{\epsilon_{z z}}\left(\bar{\nabla}_{j} g^{(n-2)}+l_{j} g^{(n-1)}\right)\right] \Phi^{(1)}\right] .
\end{aligned}
$$

Note that if all electric terms are disregarded in the relations presented in this section, the simplified expressions are in line with the work by Mauritsson et al. [35] on anisotropic elastic plates.

\section{Plate equations}

This section aims at deriving the dynamical plate equations through the surface boundary conditions at $z= \pm h$. From now on it is understood that the power series sums are to be truncated, where the truncation level could be chosen to any order. In order to write the expressions on a compact form using abbreviated subscripts, the prescribed fields for mechanical tractions and electric surface charges are here expressed in terms of mechanical stresses and electric displacements.

At each point on the surfaces $z= \pm h$, either the mechanical stress or the mechanical displacement is to be prescribed in each coordinate direction. These given fields are denoted by $\left\{T_{I}^{+}, u_{i}^{+}\right\}$ and $\left\{T_{I}^{-}, u_{i}^{-}\right\}$at the upper and lower surfaces, respectively, where $I=5,4,3$ and $i=x, y, z$. Hence, one of the fields for each of the six pairs $\left\{T_{x z}^{ \pm}, u_{x}^{ \pm}\right\},\left\{T_{y z}^{ \pm}, u_{y}^{ \pm}\right\},\left\{T_{z z}^{ \pm}, u_{z}^{ \pm}\right\}$is to be given, resulting in three mechanical boundary conditions at each point on the upper and lower surfaces, respectively. Similarly, either the normal electric displacement component or the electric potential is to be prescribed at each point on the surfaces $z= \pm h$. These are expressed as $\left\{D_{z}^{+}, \Phi^{+}\right\}$and $\left\{D_{z}^{-}, \Phi^{-}\right\}$at the upper and lower surfaces, respectively, and result in one electric boundary conditions at each point on the surfaces. All in all four boundary conditions are thus to be stated at each surface point. Using (3.1), (3.2) and (3.13), (3.14) these are written

$$
\begin{aligned}
& \sum_{n=0}^{N} h^{n} P^{(n)}(x, y, t)=P^{+}(x, y, t), \quad \sum_{n=0}^{N}(-h)^{n} P^{(n)}(x, y, t)=P^{-}(x, y, t), \\
& \sum_{n=0}^{N+1} h^{n} p^{(n)}(x, y, t)=P^{+}(x, y, t), \quad \sum_{n=0}^{N+1}(-h)^{n} p^{(n)}(x, y, t)=p^{-}(x, y, t),
\end{aligned}
$$

where $P=\left\{T_{I}, D_{z}\right\}$ and $p=\left\{u_{i}, \Phi\right\}$ for $I=5,4,3$ and $i=x, y, z$. The difference in number of terms used for these equations is due to the differences in the differential orders. Since the mechanical stress terms $T_{I}^{(n)}$ in (3.15) and the electric displacement terms $D_{i}^{(n)}$ in (3.16) include spatial derivatives of one order higher than the mechanical displacements $u_{i}^{(n)}$ and the electric potentials $\Phi^{(n)}$, an extra term is to be included in the latter sums to obtain a consistent set of plate equations.

These surface boundary conditions obtained from combinations of (4.1) and (4.2) constitute the sought hyperbolic set of eight scalar equations of motion for a piezoelectric plate. By using the expansions (3.9), (3.10) and (3.17), (3.18) a hierarchy of piezoelectric plate equations are expressed in terms of the lowest order fields $\left\{u_{x}^{(0)}, u_{y}^{(0)}, u_{z}^{(0)}, \phi^{(0)}, u_{x}^{(1)}, u_{y}^{(1)}, u_{z}^{(1)}, \phi^{(1)}\right\}$. This system may be truncated to any order where the higher order sets can be used for obtaining high accuracy solutions, while the lower order sets may be used as engineering plate equations. Note that the resulting governing set of differential equations are in the general case fundamentally different for various parts of the plate, depending on the sort of mechanical boundary conditions (stresses or displacements) and the sort of electric boundary conditions (potentials or displacements) prescribed on a specific surface region. However, the surface boundary conditions are in all cases fulfilled exactly for the expansion order in question.

Each of the eight surface boundary conditions (4.1) and (4.2) involves spatial derivatives of order $N+1$ on $u_{i}^{(0)}$ and $\Phi^{(0)}$ (except when $N=0$ for prescribed mechanical displacements and electric potentials) and spatial derivatives of order $N$ on $u_{i}^{(1)}$ and $\Phi^{(1)}$. However, the resulting system of eight plate equations turns out to be of differential order $2(4 N-1)$ in the $x y$ coordinates due to cancelation effects. This is readily seen by eliminating all but one of the fields, say $w_{0}$, resulting in one equation of spatial order $2(4 N-1)$. From this single equation, it is also seen that the number $N$ should preferably be an odd integer. By choosing $N$ odd, this equation includes space and time derivatives on $w_{0}$ up to differential order $N+1$ which render asymptotically correct results [35]. For $N$ even, the equation is only asymptotically correct up to differential order $N$. Furthermore, to account for the bending stiffness in the plate the smallest truncation order is $N=3$, as this truncation yields fourth order derivatives with respect to the time and space coordinates on $w_{0}$ in the one single equation.

\subsection{Comparisons to exact theory}

The hierarchy of approximate plate equations may be compared analytically to the exact equations of motion by studying the corresponding frequency equations. Consider here the standard case of an infinite plate where the surface boundaries are stress free with zero potential. For the present approximate theory, separate frequency equations for antisymmetric and symmetric motions are obtained as polynomial expressions in terms of the wave numbers and the frequency. These results are to be compared to the exact antisymmetric and symmetric transcendental frequency equations, where the expressions in terms of wave numbers and frequency are expanded in Maclaurin series. Although comparisons could be performed to any order, only the case with truncation order $N=5$ has been studied due to the complicated expressions. Hereby, each polynomial term up to and including order $N=5$ in the approximate theories (antisymmetric and symmetric) is identical to the corresponding term in the Maclaurin series using exact theory. This illustrates that the present approach is asymptotically correct for these low order terms, and thus probably also to arbitrary order. Similar results are reported for longitudinal displacements in rods [37], beams [38] and for flexural elastic plates $[40,44]$ when using a series expansion approach. Hence, the subsequent plate equations presented in Section 6 are thus asymptotically correct to order $N=3$ (antisymmetric) and $N=1$ (symmetric), respectively.

\section{Edge boundary conditions}

This section aims at developing the $4 N-1$ boundary conditions on each edge for a rectangular plate. This is performed in a systematic manner based on variational calculus.

Consider a rectangular plate where $-a \leqslant x \leqslant a$ and $-b \leqslant y \leqslant b$. As for the surfaces $z= \pm h$, either the mechanical stress or the mechanical displacement is to be prescribed in each coordinate direction over the entire thickness interval $-h \leqslant z \leqslant h$. At $x= \pm a$ these fields are denoted $\left\{T_{I}^{ \pm a}, u_{i}^{ \pm a}\right\}$ where $I=1,6,5$ and $i=x, y, z$. Similarly, either the normal electric displacement component or 
the electric potential is to be prescribed, which at $x= \pm a$ are denoted $\left\{D_{x}^{ \pm a}, \Phi^{ \pm a}\right\}$. The edges $y= \pm b$ are treated analogously.

The edge boundary conditions may be obtained by adopting a generalized Hamilton's principle where mechanical stresses and mechanical displacements as well as the electric displacements and electric potentials are varied simultaneously and independently. Following the results from variational calculus presented in $[37,38]$, the edge boundary conditions are obtained in a systematic manner. Since all boundary fields hereby are derived in the same manner, the procedure is here presented for a given normal stress at $x=a$. Assuming a prescribed stress $T_{x x}^{+a}(y, z, t)$, the variationally consistent boundary condition is expressed as [35]

$\int_{-h}^{h}\left(T_{x x}^{+a}(y, z, t)-\sum_{n=0}^{M} z^{n} T_{x x}^{(n)}(a, y, t)\right) z^{k} d z=0, \quad k=0,1, \ldots, M$,

adopting (3.13) to order $M$. It is thus straightforward to derive the $M+1$ unknowns $T_{x x}^{(n)}$ from the system of $M+1$ equations obtained from the performed integrals. The hereby obtained representation of the boundary stress function $T_{x x}$ in power series is identical to the expansion of the given function $T_{x x}^{+a}$ in terms of Legendre polynomials $P_{n}(z / h)$ of order $M$. Consequently

$T_{x x}^{+a}(y, z, t) \approx \sum_{n=0}^{M} a_{n}(y, t) P_{n}(z / h)=\sum_{n=0}^{M} z^{n} T_{x x, n}^{+a}(y, t)$,

and hereby $T_{x x}^{(n)}(a, y, t)=T_{x x, n}^{+a}(y, t)$. Note that the standard case $T_{x x}^{+a}=0$ results in $T_{x x}^{(n)}=0$ for all $n$.

In line with the anisotropic elastic plate, the total number of $4 N-1$ edge conditions on $x= \pm a$ are distributed as $N$ boundary conditions $(M=N-1)$ on each of the three in plane pairs $\left\{T_{x x}^{(n)}, u_{n}\right\},\left\{T_{x y}^{(n)}, v_{n}\right\},\left\{D_{x}^{(n)}, \Phi^{(n)}\right\}$ while $N-1$ boundary conditions $(M=N-2)$ on the out of plane pair $\left\{T_{x z}^{(n)}, w_{n}\right\}$. Corresponding results are obtained at $y= \pm b$. The orders, $k=0,1, \ldots$, are chosen in a manner to obtain the correct distribution of boundary conditions between the symmetric and antisymmetric parts.

In the case of connected homogeneous rectangular plates with different properties (geometrical, material, surface boundary conditions) the $2(4 N-1)$ coupling conditions at a common edge are deduced in a similar way as the edge boundary conditions see [35].

\subsection{Calculation of fields}

From the solution of the piezoelectric plate equations of motion (4.1) and (4.2) adopting the $4 N-1$ boundary conditions on each edge, the plate fields are to be calculated by truncating the power series expansions (3.1), (3.2) and (3.13), (3.14). Since a lower truncation order is used for the edge boundary conditions than for the surface boundary conditions as described above, one has to make a choice regarding the adopted number of terms. Based on experience from [35,37], the same truncation procedure is used as for the governing plate Eqs. (4.1) and (4.2); $N+2$ terms for the mechanical displacements and the electric potential in (3.1) and (3.2), while $N+1$ terms are adopted for the mechanical stresses and the electric displacements (3.13) and (3.14). Hereby the surface boundary conditions are exactly fulfilled while the edge boundary conditions are corrupted. However, close to or even at the edge boundaries, the discrepancies due to extra terms in the chosen approach are either negligible or small among the cases studied.

\section{Orthotropic piezoelectric plate poled in the $x$ direction}

The preceding set of piezoelectric plate equations with pertinent boundary conditions are expressed in a general form that are applicable to all sorts of anisotropic configurations. By choosing the truncation order, plate equations of various differential order are obtained in a very systematic manner. However, in many applications the structure properties involve symmetry planes such as for orthotropic materials. Therefore consider the special case of a material of class $2 \mathrm{~mm}$ poled in the $x$ direction, i.e. the polarization axis lies in the same plane as the plate. Such a plate configuration has been studied by Tiersten [25] which calls for comparisons between theories. This turns the recursion formulas and the plate equations to a considerably more tractable form, where it is possible to study standard boundary problems separately for antisymmetric and symmetric motions. It is straightforward to truncate the equations to arbitrary order resulting in equations of high accuracy that can be used as benchmark solutions. By taking lower order truncation, engineering piezoelectric plate equations are obtained that may be compared to other approximate theories for orthotropic piezoelectric plates.

For the material configuration in question there are now 9 independent stiffness constants

$\left[c_{I J}\right]=\left(\begin{array}{cccccc}c_{11} & c_{12} & c_{13} & 0 & 0 & 0 \\ c_{12} & c_{22} & c_{23} & 0 & 0 & 0 \\ c_{13} & c_{23} & c_{33} & 0 & 0 & 0 \\ 0 & 0 & 0 & c_{44} & 0 & 0 \\ 0 & 0 & 0 & 0 & c_{55} & 0 \\ 0 & 0 & 0 & 0 & 0 & c_{66}\end{array}\right)$,

3 independent dielectric constants

$$
\left[\epsilon_{i j}\right]=\left(\begin{array}{ccc}
\epsilon_{x x} & 0 & 0 \\
0 & \epsilon_{y y} & 0 \\
0 & 0 & \epsilon_{z z}
\end{array}\right) \text {. }
$$

and 5 independent piezoelectric coupling constants

$\left[e_{i j}\right]=\left(\begin{array}{cccccc}e_{x 1} & e_{x 2} & e_{x 3} & 0 & 0 & 0 \\ 0 & 0 & 0 & 0 & 0 & e_{y 6} \\ 0 & 0 & 0 & 0 & e_{z 5} & 0\end{array}\right)$

The recursion formulas (3.3) and (3.4) are explicitly written

$$
\begin{aligned}
u_{n+2}= & \frac{1}{(n+1)(n+2) g_{55}}\left[\epsilon_{z z} \rho \partial_{t}^{2} u_{n}-g_{11} \partial_{x}^{2} u_{n}-g_{66} \partial_{y}^{2} u_{n}\right. \\
& \left.-g_{126} \partial_{x} \partial_{y} v_{n}-(n+1) g_{135} \partial_{x} w_{n+1}-h_{15} \partial_{x}^{2} \Phi_{n}-h_{65} \partial_{y}^{2} \Phi_{n}\right], \\
v_{n+2}= & \frac{1}{(n+1)(n+2) c_{44}}\left[\rho \partial_{t}^{2} v_{n}-c_{66} \partial_{x}^{2} v_{n}-c_{22} \partial_{y}^{2} v_{n}-c_{126} \partial_{x} \partial_{y} u_{n}\right. \\
& \left.-(n+1) c_{234} \partial_{y} w_{n+1}-e_{26} \partial_{x} \partial_{y} \Phi_{n}\right], \\
w_{n+2}= & \frac{1}{(n+1)(n+2) c_{33}}\left[\rho \partial_{t}^{2} w_{n}-c_{55} \partial_{x}^{2} w_{n}-c_{44} \partial_{y}^{2} w_{n}\right. \\
& \left.-(n+1) c_{135} \partial_{x} u_{n+1}-(n+1) c_{234} \partial_{y} v_{n+1}-(n+1) e_{35} \partial_{x} \Phi_{n+1}\right], \\
\Phi_{n+2}= & \frac{1}{(n+1)(n+2) g_{55}}\left[e_{z 5} \rho \partial_{t}^{2} u_{n}-f_{11} \partial_{x}^{2} u_{n}-f_{66} \partial_{y}^{2} u_{n}\right. \\
& \left.-f_{126} \partial_{x} \partial_{y} v_{n}-(n+1) f_{13} \partial_{x} w_{n+1}-g_{51} \partial_{x}^{2} \Phi_{n}-g_{56} \partial_{y}^{2} \Phi_{n}\right],
\end{aligned}
$$

where $n=0,1,2, \ldots$, and the fields are denoted $u_{x}^{(n)}=u_{n}$, $u_{y}^{(n)}=v_{n}, u_{z}^{(n)}=w_{n}$, and $\Phi^{(n)}=\Phi_{n}$ for convenience. In line with (3.9) and (3.10) these higher order fields may be expressed in the lowest order fields, not pursued here.

Several new constants are here introduced: 


$$
\begin{array}{lll}
c_{126}=c_{12}+c_{66}, & c_{135}=c_{13}+c_{55}, & c_{234}=c_{23}+c_{44}, \\
e_{26}=e_{x 2}+e_{y 6}, & e_{35}=e_{x 3}+e_{z 5}, & \\
f_{11}=c_{11} e_{z 5}-c_{55} e_{x 1}, & f_{13}=c_{13} e_{z 5}-c_{55} e_{x 3}, & f_{66}=c_{66} e_{z 5}-c_{55} e_{y 6}, \\
f_{126}=c_{126} e_{z 5}-c_{55} e_{26}, & \\
g_{11}=c_{11} \epsilon_{z z}+e_{z 5} e_{x 1}, & g_{66}=c_{66} \epsilon_{z z}+e_{z 5} e_{y 6}, & \\
g_{51}=c_{55} \epsilon_{x x}+e_{z 5} e_{x 1}, & g_{56}=c_{55} \epsilon_{y y}+e_{z 5} e_{y 6}, & g_{55}=c_{55} \epsilon_{z z}+e_{z 5}^{2}, \\
g_{126}=c_{126} \epsilon_{z z}+e_{z 5} e_{26}, & g_{135}=c_{135} \epsilon_{z z}+e_{z 5} e_{35}, & \\
h_{15}=e_{x 1} \epsilon_{z z}-e_{z 5} \epsilon_{x x}, & h_{65}=e_{y 6} \epsilon_{z z}-e_{z 5} \epsilon_{y y} .
\end{array}
$$

The mechanical stress expansion functions $T_{i j}^{(n)}$ are obtained from (3.15) and these become

$$
\begin{aligned}
T_{x x}^{(n)} & =c_{11} \partial_{x} u_{n}+c_{12} \partial_{y} v_{n}+(n+1) c_{13} w_{n+1}+e_{x 1} \partial_{x} \Phi_{n}, \\
T_{y y}^{(n)} & =c_{12} \partial_{x} u_{n}+c_{22} \partial_{y} v_{n}+(n+1) c_{23} w_{n+1}+e_{x 2} \partial_{x} \Phi_{n}, \\
T_{z z}^{(n)} & =c_{13} \partial_{x} u_{n}+c_{23} \partial_{y} v_{n}+(n+1) c_{33} w_{n+1}+e_{x 3} \partial_{x} \Phi_{n}, \\
T_{x z}^{(n)} & =c_{55}\left[(n+1) u_{n+1}+\partial_{x} w_{n}\right]+(n+1) e_{z 5} \Phi_{n+1}, \\
T_{y z}^{(n)} & =c_{44}\left[(n+1) v_{n+1}+\partial_{y} w_{n}\right], \\
T_{x y}^{(n)} & =c_{66}\left(\partial_{y} u_{n}+\partial_{x} v_{n}\right)+e_{y 6} \partial_{y} \Phi_{n} .
\end{aligned}
$$

Similarly, the electric displacements functions $D_{i}^{(n)}$ are obtained from (3.16)

$$
\begin{aligned}
& D_{x}^{(n)}=e_{x 1} \partial_{x} u_{n}+e_{x 2} \partial_{y} v_{n}+(n+1) e_{x 3} w_{n+1}-\epsilon_{x x} \partial_{x} \Phi_{n}, \\
& D_{y}^{(n)}=e_{y 6}\left(\partial_{y} u_{n}+\partial_{x} v_{n}\right)-\epsilon_{y y} \partial_{y} \Phi_{n}, \\
& D_{z}^{(n)}=e_{z 5} \partial_{x} w_{n}+(n+1) e_{z 5} u_{n+1}-(n+1) \epsilon_{z z} \Phi_{n+1} .
\end{aligned}
$$

The fields in (6.6) and (6.7) may be written in terms of the lowest order fields as in (3.17) and (3.18) using (6.4).

As noted before various combinations of the boundary conditions stated in (4.1) and (4.2) may be used. Consider for simplicity the case when either the mechanical stress or the mechanical displacement is prescribed on both the upper and lower surfaces for a specific direction. Similarly, assume that either the electric potential or the electric displacement is prescribed on both the upper and lower surfaces. By adding and subtracting the boundary conditions in (4.1) and (4.2), equations containing only even or odd expansions in $h$ are obtained according to

$$
\begin{aligned}
& \sum_{n=0}^{\lfloor N / 2\rfloor} h^{2 n} P^{(2 n)}=\frac{1}{2}\left(P^{+}+P^{-}\right), \quad \sum_{n=0}^{\lfloor(N-1) / 2\rfloor} h^{2 n+1} P^{(2 n+1)}=\frac{1}{2}\left(P^{+}-P^{-}\right), \\
& \sum_{n=0}^{\lfloor N+1) / 2\rfloor} h^{2 n} p^{(2 n)}=\frac{1}{2}\left(p^{+}+p^{-}\right), \quad \sum_{n=0}^{\lfloor N / 2\rfloor} h^{2 n+1} p^{(2 n+1)}=\frac{1}{2}\left(p^{+}-p^{-}\right),
\end{aligned}
$$

where as before $P=\left\{T_{x z}, T_{y z}, T_{z z}, D_{z}\right\}$ and $p=\{u, v, w, \Phi\}$. Here $\lfloor n\rfloor$ denotes the floor of $n$, i.e. the nearest lower integer to $n$. These equations constitute decoupled sets corresponding to symmetrical and antisymmetrical motions. The symmetric part uses the first relation in (6.8) for $T_{z z}$ and the second relation in (6.8) for $\left\{T_{x z}, T_{y z}, D_{z}\right\}$, and the first relation in (6.9) for $\{u, v, \Phi\}$ and the second relation in (6.9) for $w$. Hence, all possible combinations of these symmetric parts of the boundary conditions involve only the fields $\left\{u_{0}, v_{0}, w_{1}, \Phi_{0}\right\}$. Naturally the complementary combinations correspond to antisymmetrical sets involving $\left\{u_{1}, v_{1}, w_{0}, \Phi_{1}\right\}$.

For the sake of clarity, the plate equations of order $N=3$ are presented explicitly below for antisymmetric motions while equations of order $N=1$ are presented for symmetric motions. Hereby the antisymmetric motions correctly account for the flexural effects through the $h^{3}$ terms. For the mechanical surface boundary conditions, only the traditional case for prescribed stresses $T_{I}^{ \pm}$are presented. The mechanical displacement boundary conditions are easily treated using (6.9) together with the recursion relations
(6.4). As for the electrical surface boundary conditions, both types are given since potential boundary conditions $\Phi^{ \pm}$are usually stated for actuator problems while electric displacement boundary conditions $D_{z}^{ \pm}$are usually stated for sensor problems. Concerning the edge boundary conditions, all combinations of mechanical and electrical fields are presented.

\subsection{Antisymmetric motion $N=3$}

The plate equations for prescribed surface stresses are expressed in line with (6.8) using (6.6) and (6.4) as

$$
\begin{aligned}
c_{55}\left(u_{1}+\partial_{x} w_{0}\right) & +e_{z 5} \Phi_{1}+\frac{h^{2}}{2 c_{33}}\left[c_{33} \rho \partial_{t}^{2} u_{1}-\left(c_{11} c_{33}-c_{13} c_{135}\right) \partial_{x}^{2} u_{1}\right. \\
& -c_{33} c_{66} \partial_{y}^{2} u_{1}-\left(c_{33} c_{126}-c_{13} c_{234}\right) \partial_{x} \partial_{y} v_{1}-c_{13} \rho \partial_{t}^{2} \partial_{x} w_{0} \\
& +c_{13} c_{55} \partial_{x}^{3} w_{0}+c_{13} c_{44} \partial_{x} \partial_{y}^{2} w_{0}-\left(c_{33} e_{x 1}-c_{13} e_{35}\right) \partial_{x}^{2} \Phi_{1} \\
& \left.-c_{33} e_{y 6} \partial_{y}^{2} \Phi_{1}\right]=\frac{1}{2}\left(T_{x z}^{+}+T_{x z}^{-}\right), \\
c_{44}\left(v_{1}+\partial_{y} w_{0}\right) & +\frac{h^{2}}{2 c_{33}}\left[c_{33} \rho \partial_{t}^{2} v_{1}-\left(c_{22} c_{33}-c_{23} c_{234}\right) \partial_{y}^{2} v_{1}-c_{33} c_{66} \partial_{x}^{2} v_{1}\right. \\
& -\left(c_{33} c_{126}-c_{23} c_{135}\right) \partial_{x} \partial_{y} u_{1}-c_{23} \rho \partial_{t}^{2} \partial_{y} w_{0}+c_{23} c_{44} \partial_{y}^{3} w_{0} \\
& \left.+c_{23} c_{55} \partial_{x}^{2} \partial_{y} w_{0}-\left(c_{33} e_{26}-c_{23} e_{35}\right) \partial_{x} \partial_{y} \Phi_{1}\right]=\frac{1}{2}\left(T_{y z}^{+}+T_{y z}^{-}\right)
\end{aligned}
$$

$$
\begin{aligned}
& h\left[\rho \partial_{t}^{2} w_{0}-c_{55}\left(\partial_{x} u_{1}+\partial_{x}^{2} w_{0}\right)-c_{44}\left(\partial_{y} v_{1}+\partial_{y}^{2} w_{0}\right)-e_{z 5} \partial_{x} \Phi_{1}\right] \\
& \quad+\frac{h^{3}}{6 c_{33}}\left[\rho^{2} \partial_{t}^{4} w_{0}-c_{13} c_{55} \partial_{x}^{4} w_{0}-c_{23} c_{44} \partial_{y}^{4} w_{0}-\left(c_{13} c_{44}+c_{23} c_{55}\right) \partial_{x}^{2} \partial_{y}^{2} w_{0}\right. \\
& -\left(c_{55}-c_{13}\right) \rho \partial_{t}^{2} \partial_{x}^{2} w_{0}-\left(c_{44}-c_{23}\right) \rho \partial_{t}^{2} \partial_{y}^{2} w_{0}-\left(c_{33}+c_{135}\right) \rho \partial_{t}^{2} \partial_{x} u_{1} \\
& +\left(c_{11} c_{33}-c_{13} c_{135}\right) \partial_{x}^{3} u_{1}+\left[c_{33}\left(c_{126}+c_{66}\right)-c_{23} c_{135}\right] \partial_{x} \partial_{y}^{2} u_{1} \\
& -\left(c_{33}+c_{234}\right) \rho \partial_{t}^{2} \partial_{y} v_{1}+\left(c_{22} c_{33}-c_{23} c_{234}\right) \partial_{y}^{3} v_{1}+\left[c_{33}\left(c_{126}+c_{66}\right)\right. \\
& \left.-c_{13} c_{234}\right] \partial_{x}^{2} \partial_{y} v_{1}-e_{35} \rho \partial_{x} \partial_{t}^{2} \Phi_{1}+\left(c_{33} e_{x 1}-c_{13} e_{35}\right) \partial_{x}^{3} \Phi_{1} \\
& \left.\quad+\left[c_{33}\left(e_{26}+e_{y 6}\right)-c_{23} e_{35}\right] \partial_{x} \partial_{y}^{2} \Phi_{1}\right]=\frac{1}{2}\left(T_{z z}^{+}-T_{z z}^{-}\right) .
\end{aligned}
$$

For prescribed electric potential or electric displacement, the corresponding equations read

$$
\begin{aligned}
h \Phi_{1} & +\frac{h^{3}}{6 c_{33} g_{55}}\left[c_{33} e_{z 5} \rho \partial_{t}^{2} u_{1}-\left(c_{33} f_{11}-c_{135} f_{13}\right) \partial_{x}^{2} u_{1}-c_{33} f_{66} \partial_{y}^{2} u_{1}\right. \\
& -\left(c_{33} f_{126}-c_{234} f_{13}\right) \partial_{x} \partial_{y} v_{1}-f_{13} \rho \partial_{x} \partial_{t}^{2} w_{0}+c_{55} f_{13} \partial_{x}^{3} w_{0} \\
& \left.+c_{44} f_{13} \partial_{x} \partial_{y}^{2} w_{0}-\left(c_{33} g_{51}-e_{35} f_{13}\right) \partial_{x}^{2} \Phi_{1}-c_{33} g_{56} \partial_{y}^{2} \Phi_{1}\right] \\
& =\frac{1}{2}\left(\Phi^{+}-\Phi^{-}\right)
\end{aligned}
$$

$$
\begin{aligned}
e_{z 5}\left(u_{1}+\partial_{x} w_{0}\right) & -\epsilon_{z z} \Phi_{1}+\frac{h^{2}}{2 c_{33}}\left[-\left(c_{33} e_{x 1}-c_{135} e_{x 3}\right) \partial_{x}^{2} u_{1}-c_{33} e_{y 6} \partial_{y}^{2} u_{1}\right. \\
& -\left(c_{33} e_{z 6}-c_{234} e_{x 3}\right) \partial_{x} \partial_{y} v_{1}-e_{x 3} \rho \partial_{t}^{2} \partial_{x} w_{0}+c_{55} e_{x 3} \partial_{x}^{3} w_{0} \\
& \left.+c_{44} e_{x 3} \partial_{x} \partial_{y}^{2} w_{0}+\left(e_{x 3} e_{35}+c_{33} \epsilon_{x x}\right) \partial_{x}^{2} \Phi_{1}+c_{33} \epsilon_{y y} \partial_{y}^{2} \Phi_{1}\right] \\
& =\frac{1}{2}\left(D_{z}^{+}+D_{z}^{-}\right) .
\end{aligned}
$$

In order to solve for $\left\{u_{1}, v_{1}, w_{0}, \Phi_{1}\right\}$ from the set of four plate equations, (6.10)-(6.12) together with (6.13) or (6.14), the pertinent set of four boundary conditions at each edge is to be stated as described in Section 5. Consider the edge $x=a$, the boundary conditions for prescribed displacements and potential become

$u_{1}^{+a}(y, t)=u_{1}, \quad v_{1}^{+a}(y, t)=v_{1}, \quad w_{0}^{+a}(y, t)=w_{0}, \quad \Phi_{1}^{+a}(y, t)=\Phi_{1}$, 
following the notation indicated in (5.2) using Legendre polynomial expansions of $\left\{u^{+a}(y, z, t), v^{+a}(y, z, t), w^{+a}(y, z, t), \Phi^{+a}(y, z, t)\right\}$. As for prescribed stresses and electric displacement, these boundary conditions become

$$
\begin{aligned}
T_{x x, 1}^{+a}(y, t)= & \left(c_{11}-\beta_{13} c_{135}\right) \partial_{x} u_{1}+\left(c_{12}-\beta_{13} c_{234}\right) \partial_{y} v_{1}+\beta_{13} \rho \partial_{t}^{2} w_{0} \\
& -\beta_{13} c_{55} \partial_{x}^{2} w_{0}-\beta_{13} c_{44} \partial_{y}^{2} w_{0}+\left(e_{x 1}-\beta_{13} e_{35}\right) \partial_{x} \Phi_{1}, \\
T_{x y, 1}^{+a}(y, t)= & c_{66}\left(\partial_{y} u_{1}+\partial_{x} v_{1}\right)+e_{y 6} \partial_{y} \Phi_{1} \\
T_{x z, 0}^{+a}(y, t)= & c_{55}\left(u_{1}+\partial_{x} w_{0}\right)+e_{z 5} \Phi_{1}, \\
D_{x x, 1}^{+a}(y, t)= & \left(e_{x 1}-\beta_{135} e_{x 3}\right) \partial_{x} u_{1}+\left(e_{x 2}-\beta_{234} e_{x 3}\right) \partial_{y} v_{1}+\alpha \rho \partial_{t}^{2} w_{0} \\
& -\beta_{55} e_{x 3} \partial_{x}^{2} w_{0}-\beta_{44} e_{x 3} \partial_{y}^{2} w_{0}-\left(\epsilon_{x x}+\alpha e_{35}\right) \partial_{x} \Phi_{1},
\end{aligned}
$$

where (6.6) and (6.7), the recursion relations (6.4) and the Legendre expansion (5.2) have been used. Naturally similar results are obtained for $x=-a$. For the edge $y=b$, the boundary conditions for prescribed displacements and potential become like in (6.15), while for prescribed stresses and electric displacement one has

$T_{y y, 1}^{+b}(x, t)=\left(c_{12}-\beta_{23} c_{135}\right) \partial_{x} u_{1}+\left(c_{22}-\beta_{23} c_{234}\right) \partial_{y} v_{1}+\beta_{23} \rho \partial_{t}^{2} w_{0}$

$-\beta_{23} c_{55} \partial_{x}^{2} w_{0}-\beta_{23} c_{44} \partial_{y}^{2} w_{0}+\left(e_{x 2}-\beta_{23} e_{35}\right) \partial_{x} \Phi_{1}$,

$T_{x y, 1}^{+b}(x, t)=c_{66}\left(\partial_{y} u_{1}+\partial_{x} v_{1}\right)+e_{y 6} \partial_{y} \Phi_{1}$,

$T_{y z, 0}^{+b}(x, t)=c_{44}\left(v_{1}+\partial_{y} w_{0}\right)$,

$D_{y y, 1}^{+b}(x, t)=e_{y 6}\left(\partial_{y} u_{1}+\partial_{x} v_{1}\right)-\epsilon_{y y} \partial_{y} \Phi_{1}$

In (6.16) and (6.17) the following new constants are introduced

$\alpha=e_{x 3} / c_{33}, \quad \beta_{i j}=c_{i j} / c_{33}, \quad \beta_{i j k}=c_{i j k} / c_{33}$.

\subsection{Symmetric motion $N=1$} (6.8)

The plate equations for prescribed surface stresses become from

$$
\begin{gathered}
h\left[\rho \partial_{t}^{2} u_{0}-c_{11} \partial_{x}^{2} u_{0}-c_{66} \partial_{y}^{2} u_{0}-c_{126} \partial_{x} \partial_{y} v_{0}-c_{13} \partial_{x} w_{1}\right. \\
\left.-e_{x 1} \partial_{x}^{2} \Phi_{0}-e_{y 6} \partial_{y}^{2} \Phi_{0}\right]=\frac{1}{2}\left(T_{x z}^{+}-T_{x z}^{-}\right),
\end{gathered}
$$

$$
\begin{aligned}
& h\left[\rho \partial_{t}^{2} v_{0}-c_{22} \partial_{y}^{2} v_{0}-c_{66} \partial_{x}^{2} v_{0}-c_{126} \partial_{x} \partial_{y} u_{0}-c_{23} \partial_{y} w_{1}-e_{26} \partial_{x} \partial_{y} \Phi_{0}\right] \\
& \quad=\frac{1}{2}\left(T_{y z}^{+}-T_{y z}^{-}\right)
\end{aligned}
$$

$c_{33} w_{1}+c_{13} \partial_{x} u_{0}+c_{23} \partial_{y} v_{0}+e_{x 3} \partial_{x} \Phi_{0}=\frac{1}{2}\left(T_{z z}^{+}+T_{z z}^{-}\right)$.

For prescribed electric potential or electric displacement, these equations are

$$
\begin{aligned}
& \Phi_{0}+\frac{h^{2}}{2 g_{55}}\left[e_{z 5} \rho \partial_{t}^{2} u_{0}-f_{11} \partial_{x}^{2} u_{0}-f_{66} \partial_{y}^{2} u_{0}-\left(c_{126} e_{z 5}-c_{55} e_{26}\right) \partial_{x} \partial_{y} v_{0}\right. \\
& \left.-g_{51} \partial_{x}^{2} \Phi_{0}-g_{56} \partial_{y}^{2} \Phi_{0}-f_{13} \partial_{x} w_{1}\right]=\frac{1}{2}\left(\Phi^{+}+\Phi^{-}\right) . \\
& h\left[-e_{x 1} \partial_{x}^{2} u_{0}-e_{y 6} \partial_{y}^{2} u_{0}-e_{z 6} \partial_{x} \partial_{y} v_{0}-e_{x 3} \partial_{x} w_{1}+\epsilon_{x x} \partial_{x}^{2} \Phi_{0}+\epsilon_{y y} \partial_{y}^{2} \Phi_{0}\right] \\
& \quad=\frac{1}{2}\left(D_{z}^{+}-D_{z}^{-}\right) .
\end{aligned}
$$

There are three boundary conditions needed at each edge to solve for $\left\{u_{0}, v_{0}, w_{1}, \Phi_{0}\right\}$. Consider the edge $x=a$, the boundary conditions for prescribed displacements and potential become

$u_{0}^{+a}(y, t)=u_{0}, \quad v_{0}^{+a}(y, t)=v_{0}, \quad \Phi_{0}^{+a}(y, t)=\Phi_{0}$,

while the stress and electric displacement boundary conditions are

$$
\begin{aligned}
& T_{x x, 0}^{+a}(y, t)=c_{11} \partial_{x} u_{0}+c_{12} \partial_{y} v_{0}+c_{13} w_{1}+e_{x 1} \partial_{x} \Phi_{0}, \\
& T_{x y, 0}^{+a}(y, t)=c_{66}\left(\partial_{y} u_{0}+\partial_{x} v_{0}\right)+e_{y 6} \partial_{y} \Phi_{0}, \\
& D_{x x, 0}^{+a}(y, t)=e_{x 1} \partial_{x} u_{0}+e_{x 2} \partial_{y} v_{0}+e_{x 3} w_{1}-\epsilon_{x x} \partial_{x} \Phi_{0} .
\end{aligned}
$$

At the edge $y=b$, displacement and potential boundary conditions become like in (6.24), while for prescribed stresses and electric displacement one has

$$
\begin{aligned}
& T_{y y, 0}^{+b}(x, t)=c_{12} \partial_{x} u_{0}+c_{22} \partial_{y} v_{0}+c_{23} w_{1}+e_{x 2} \partial_{x} \Phi_{0}, \\
& T_{x y, 0}^{+b}(x, t)=c_{66}\left(\partial_{y} u_{0}+\partial_{x} v_{0}\right)+e_{y 6} \partial_{y} \Phi_{0}, \\
& D_{y y, 0}^{+b}(x, t)=e_{y 6}\left(\partial_{y} u_{0}+\partial_{x} v_{0}\right)-\epsilon_{y y} \partial_{y} \Phi_{0} .
\end{aligned}
$$

Note that all the results in this section are identical to the elastic orthotropic case in [35] if the electric coupling is disregarded.

These plate equations may be compared to other theories such as those of Kirchhoff and Mindlin type [25]. For the Kirchhoff theory only a few terms are similar to the present theory, while the Mindlin equations (involving two different correction factors) resemble the asymptotic plate equations in many respects. The low order derivative terms are similar in most cases, while the present theory involves higher order derivative terms that are not part of the Mindlin theory. However, similar higher order derivative terms are found in higher order theories as given analytically in $[12,13,46]$, albeit the coefficients of these terms differ in most cases.

\section{Numerical results}

To investigate the accuracy of the present plate equations for both lower order engineering theories and the higher order sets, several numerical results are presented. The branches from the frequency equations (dispersion relations) for waves in an infinite plate poled in the $x$ direction are illustrated, where distribution plots are presented for the displacements, stresses and electric potential for the lowest antisymmetric modes. Antisymmetric and symmetric eigenfrequencies are calculated for finite square simply supported plates poled in either the $x$ or $z$ directions. Moreover, eigenfrequencies for simply supported laminated piezoelectric plates are presented. The comparisons are made with exact three dimensional theory and other approximate theories presented in the literature.

\subsection{Piezoelectric plate poled in the $x$ direction}

The piezoelectric plate is taken to be PZT-2 (Lead Zirconate Titanate) with $x$ as the polarization axis. This is a $6 \mathrm{~mm}$ material (a special case of $2 \mathrm{~mm}$ ) and elastically $y z$ is a plane of isotropy. The material constants are (Auld [45]) $c_{11}=113 \mathrm{GPa}, c_{12}=c_{13}=$ $68.1 \mathrm{GPa}, c_{22}=c_{33}=135 \mathrm{GPa}, c_{23}=67.0 \mathrm{GPa}, c_{55}=c_{66}=22.2 \mathrm{GPa}$, $e_{x 1}=9.0 \mathrm{C} / \mathrm{m}^{2}, \quad e_{x 2}=e_{x 3}=-1.9 \mathrm{C} / \mathrm{m}^{2}, \quad e_{y 6}=e_{z 5}=9.8 \mathrm{C} / \mathrm{m}^{2}, \quad \epsilon_{x x}=$ $260 \epsilon_{0}$, and $\epsilon_{y y}=\epsilon_{z z}=504 \epsilon_{0}$. Here $\epsilon_{0}=8.854 \cdot 10^{-12} \mathrm{C} / \mathrm{Vm}$ is the dielectric permittivity of free space. With $y z$ as the plane of isotropy $c_{44}=\frac{1}{2}\left(c_{22}-c_{23}\right)$. With this choice of material parameters the correction factors used in the Mindlin theory become $\kappa_{4}^{2}=\pi^{2} / 12$ and $\kappa_{5}^{2}=0.904$ [25]. The conditions are time harmonic with the time factor $\mathrm{e}^{-\mathrm{i} \omega t}$ and the dimensionless variable $\Omega=\omega h \sqrt{\rho / c_{33}}$ is used to measure the frequency. Consider free waves propagating in a direction $45^{\circ}$ to the $x$ axis. The piezoelectric layer is taken to be short-circuited $(\Delta V=0)$ and the electric potential in the middle of the plate is put to zero. The wave number in the direction of propagation is $k$.

In Fig. 2 the three first antisymmetric dispersion curves are plotted as dimensionless frequency $\Omega$ versus dimensionless wave number $k h$. The results from the asymptotic plate Eqs. (6.10)(6.13) for $N=3$ are compared with the exact theory, the Mindlin 
theory and the Kirchhoff theory [25]. The first curve corresponds to the first bending mode with an almost parabolic behavior at low frequencies. All three plate theories approximate this curve quite well for low wave numbers, but for higher wave numbers the Kirchhoff theory is not that accurate as expected. The Mindlin theory approximates the first mode slightly better than the asymptotic plate theory. The other two modes, not modeled by the Kirchhoff theory, correspond to coupled P/SV (pressure/transverse shear) and the SH (horizontal shear) modes [45].

The second set of curves correspond to a pseudo-SH mode where the Mindlin and the present $N=3$ theories give accurate approximations, although the Mindlin theory is better and predicts the cut-off frequency exactly. The third set of curves correspond to the second bending mode. Also this mode is approximated better by the Mindlin theory, at least for lower frequencies where the cut-off frequency is exactly predicted. From the dispersion curves it seems like the Mindlin theory generally gives more accurate results than the asymptotic plate theory of order $N=3$. However, it should be noticed that the correction factors used in the Mindlin theory are chosen to obtain accurate approximations of the dispersion curves at low frequencies. This does not necessarily mean that the displacements and stresses are accurately predicted, which will be seen below. Furthermore, the asymptotic plate theory converges very quickly towards the exact solution if terms of higher order are taken into account. By choosing $N=5$ in the asymptotic plate theory the approximate dispersion curves are considerably more accurate, being very close to the exact curves (not illustrated here). For $N=7$ the approximate dispersion curves become indistinguishable from the exact ones for the frequencies and wave numbers shown in Fig. 2. This feature also holds for the remaining plots Figs. 3-10.

Now consider a specific frequency $\Omega=0.5$. According to the dispersion curves in Fig. 2 there is only one real solution to the wave number for this frequency ( $k h \approx 1.44$ according to exact theory) and this solution corresponds to the first bending mode. The displacements, the electric potential and the stresses for the different theories are plotted as functions of the dimensionless thickness coordinate $z / h$ in Figs. 3-6. The figures show that the electric potential, the displacements and the stresses are not very well approximated by the Kirchhoff theory, except for the vertical normal stress $T_{z z}$, which is quite well approximated. The Mindlin theory gives more accurate results for many of the fields, but actually poorer results for the vertical normal stress. The asymptotic plate theory renders much more accurate results for all fields, especially for the electric potential $\Phi$, the vertical displacement $w$ and the shear stresses $T_{x z}$. Here, the error in sign for $\Phi$ using Kirchhoff and Mindlin theories is of particular interest. However, similar behavior is also reported for the asymptotic theory in the case of piezoelectric layer on an elastic plate [42]. Note that both the Kirchhoff and the Mindlin theories assume constant vertical displacements, lying on top of the horizontal axis in Fig. 4(b). It should also be noticed that the boundary conditions for the vertical normal stress and the electric potential at the upper surface of the plate are not fulfilled by the Mindlin or the Kirchhoff theory, but are exactly fulfilled by the asymptotic plate theory. As the results for $\left\{v, T_{y y}, T_{y z}\right\}$ resemble $\left\{u, T_{x x}, T_{x z}\right\}$ the former fields are not illustrated.

Note that for the Mindlin and the Kirchhoff theory the stresses $T_{x x}, T_{y y}$ and $T_{x y}$ are determined from the constitutive Eq. (2.5) (the direct method), while the stresses in the vertical direction $T_{z z}, T_{y z}$ and $T_{x z}$ are obtained using the indirect method. This is accomplished by inserting the stresses and displacements from the direct method into the equations of motion (2.1) and integrating with respect to $z$. The integration constants are determined by requiring that the shear stresses, $T_{y z}$ and $T_{x z}$, are zero at the plate surfaces $(z= \pm h)$ and that the normal stress, $T_{z z}$, is zero in the middle of

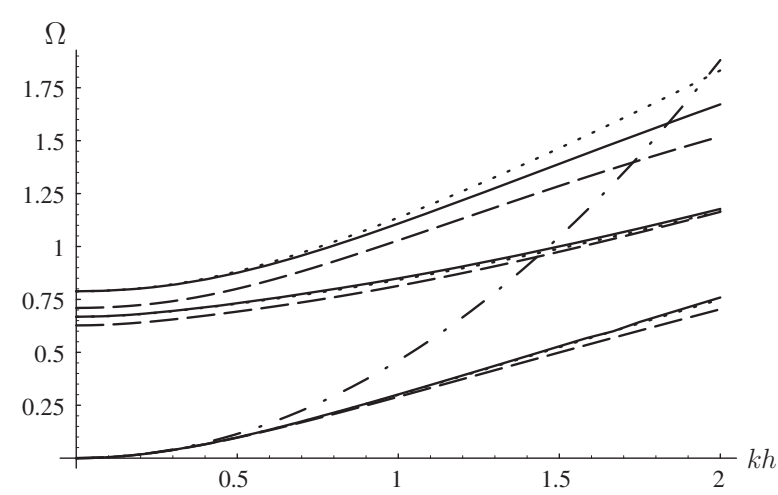

Fig. 2. Dispersion curves: - Exact, $---N=3, \cdots$ Mindlin, $-\cdot-$ Kirchhoff.

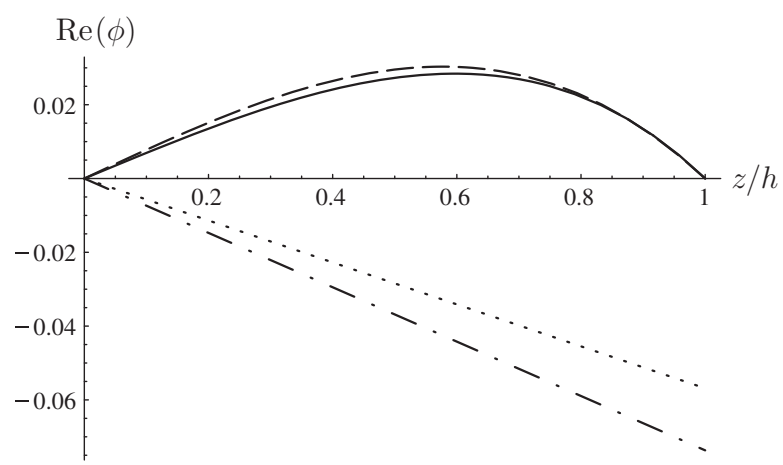

Fig. 3. Electric potential for the first bending mode, $\Omega=0.5$ : - Exact, $---N=3$, . Mindlin, -.- Kirchhoff.

the plate $(z=0)$ due to antisymmetry. This indirect way of calculating the stresses, also used by Reddy [47], gives more accurate results compared to calculating all stress components from the constitutive equations, which only yields constant or linear variations in the thickness coordinate. If the indirect method is used for the stresses using the asymptotic plate theory, the same results are obtained after truncation as with the direct method. Either the real or the imaginary part of each quantity is shown in the figures as only one of them differs from zero. Moreover, due to symmetry/ antisymmetry, only the upper half of the plate $(0 \leqslant z \leqslant h)$ is shown. The fields are normalized so that the vertical displacement in the middle of the plate equals unity, $w(z=0)=1$. The electric potential is measured with the dimensionless variable $\phi=\epsilon_{z z} /\left(h e_{z 5}\right) \Phi$ and the stresses are normalized with the stiffness constant $c_{33}$.

Consider also the frequency $\Omega=1$. According to the dispersion curves, there are three real solutions to the wave number for this frequency. In addition to the first bending mode solution there is also one solution for the pseudo-SH mode $(k h \approx 1.50)$ and one for the second bending mode $(k h \approx 0.79)$. Some of the fields for the latter two of these modes are plotted in Figs. 7-10, showing the electric potential, the dominating horizontal displacement, the vertical normal stress and the dominating shear stress for the two cases. The fields are normalized so that the imaginary part of the dominating horizontal displacement ( $u$ and $v$, respectively) equals unity at the upper surface of the plate. Also here, the asymptotic plate theory gives more accurate results than the Mindlin theory, which is not accurate at all for the electric potential or any of the stresses shown in the figures. However, it should be mentioned that the way of normalizing may affect the impression of how well different fields are approximated by different theories. In addition, if the truncation order is increased a few levels, the asymptotic 


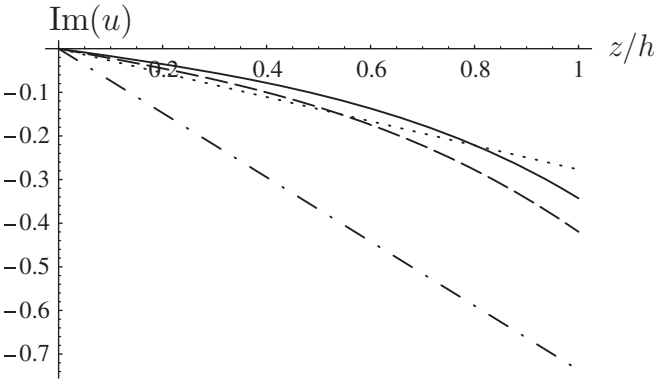

(a) Horizontal displacement in the $x$ direction.

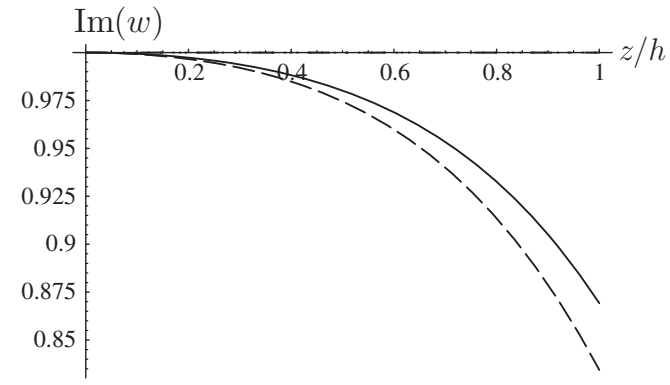

(b) Vertical displacement in the $z$ direction.

Fig. 4. Horizontal displacements for the first bending mode, $\Omega=0.5$ : - Exact, $---N=3, \cdots$ Mindlin, $-\cdot-$ Kirchhoff.

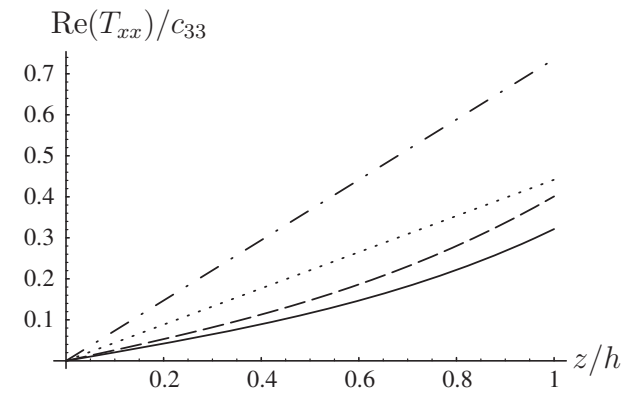

(a) Normal stress in the $x$ direction.

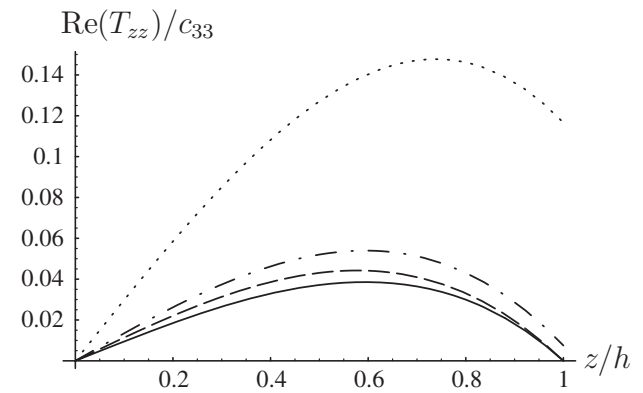

(b) Normal stress in the $z$ direction.

Fig. 5. Stresses for the first bending mode, $\Omega=0.5$ : - Exact, $---N=3, \cdots$ Mindlin, $-\cdot-$ Kirchhoff.

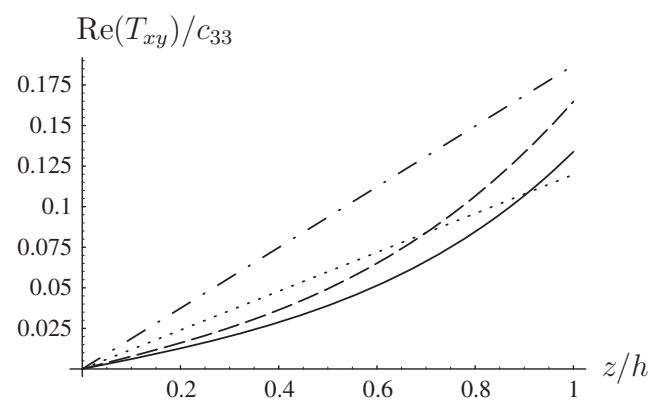

(a) Shear stress $T_{x y}$.

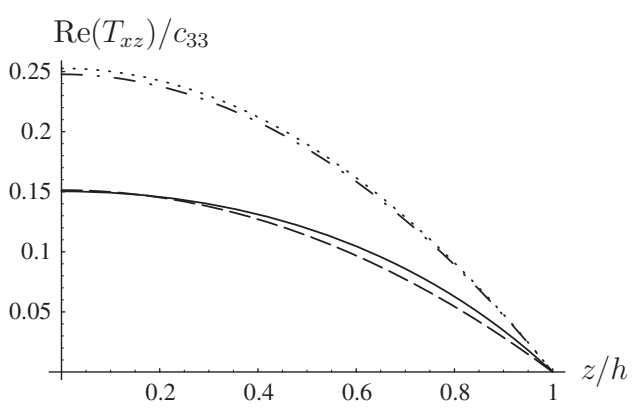

(b) Shear stress $T_{x z}$.

Fig. 6. Stresses for the first bending mode, $\Omega=0.5$ : - Exact, $---N=3, \cdots$ Mindlin, $-\cdot-$ Kirchhoff.

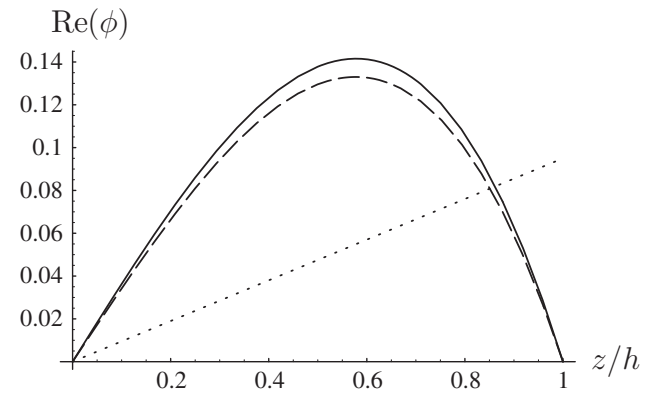

(a) Electric potential.

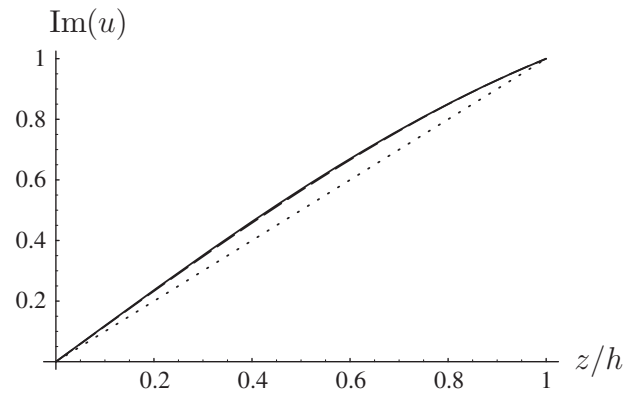

(b) Horizontal displacement in the $x$ direction.

Fig. 7. Electric potential and displacement for the pseudo-SH mode, $\Omega=1$ : - Exact, $---N=3, \cdots$ Mindlin. 


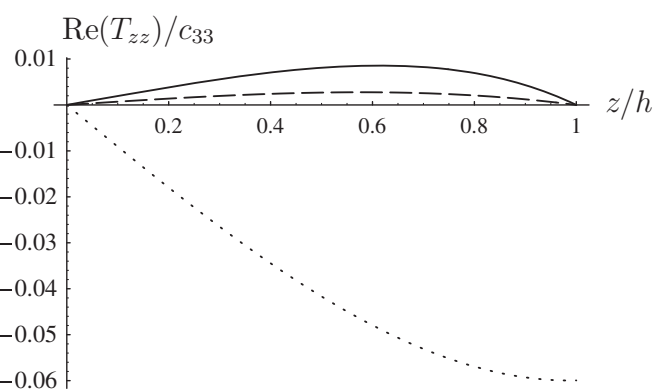

(a) Normal stress in the $z$ direction.

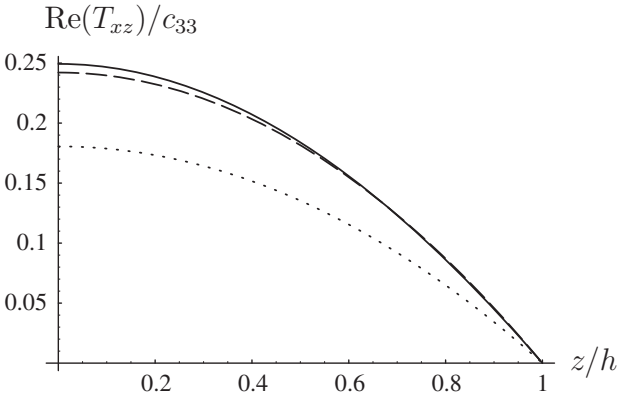

(b) Shear stress $T_{x z}$.

Fig. 8. Stresses for the pseudo-SH mode, $\Omega=1$ : Exact, $---N=3, \cdots$ Mindlin.

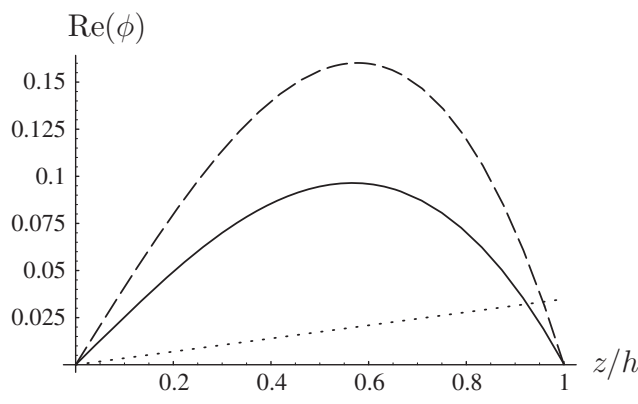

(a) Electric potential.

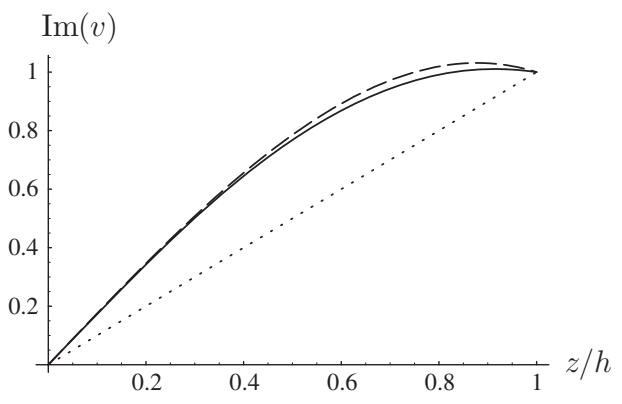

(b) Horizontal displacement in the $y$ direction.

Fig. 9. Electric potential and displacement for the second bending mode, $\Omega=1$ : - Exact, $---N=3, \cdots$ Mindlin.

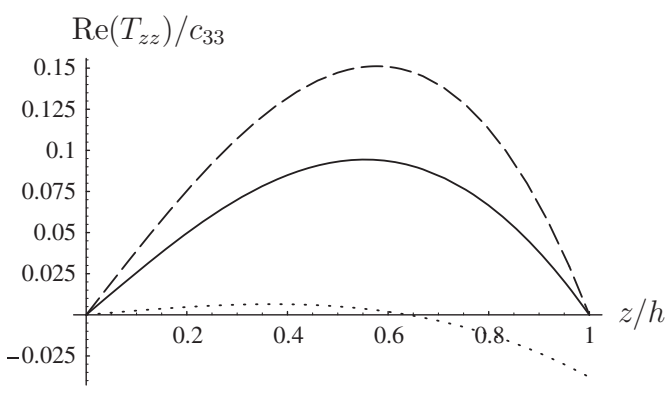

(a) Normal stress in the $z$ direction.

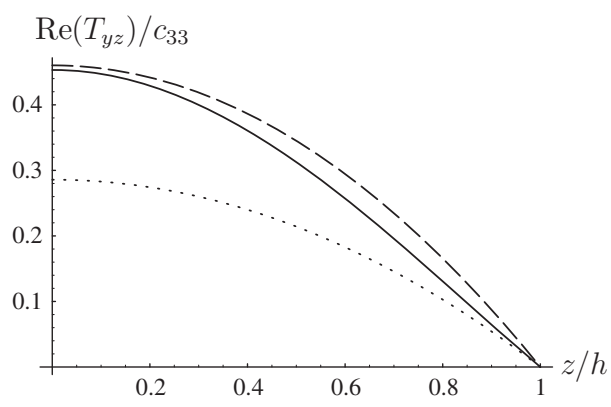

(b) Shear stress $T_{y z}$.

Fig. 10. Stresses for the second bending mode, $\Omega=1$ : - Exact, $---N=3, \cdots$ Mindlin.

plate theory curves become indistinguishable from the exact ones for all the displayed plots.

In order to further illustrate the present theory, the eigenfrequencies for simply supported quadratic plates are calculated. For exact and series expansion theories, this corresponds to mixed boundary conditions at the edges where normal stresses as well as vertical and tangential displacements are zero. Due to the polarization in the $x$ direction, the electric field is such that the normal electric field displacement is zero at $x= \pm a$ while the potential is zero at $y= \pm b$. The Tables $1-4$ illustrate the three lowest eigenfrequencies $\Omega_{i}$ for short-circuited antisymmetric and symmetric motions, respectively. Here three different plate thicknesses $a / h$ are studied for two different mode cases, where $m$ and $n$ refer to the mode numbers in the $x$ and $y$ directions, respectively.

Consider first the antisymmetric cases presented in Tables 1 and 2 . It is clear that the series expansion results converge to the exact results as the power series orders are increased. The convergence is quite rapid considering the thick plates studied. The accuracies are higher for lower frequencies as expected, while the plate thickness itself does not much influence the rate of convergence. The Kirchhoff theory models the first eigenfrequency, where the accuracy highly depends on the plate thickness. The Mindlin theory is astonishingly accurate, at least for the lower frequencies. As noted before for the dispersion curves, the Mindlin theory involves the shear correction factors that are chosen so as to give good correlations in the low frequency interval. When compared to the $N=3$ theory, the Mindlin results are generally superior for the case $m=1$ and $n=1$; especially at lower frequencies. For $m=1$ and $n=2$ the accuracy pattern is less apparent among the two theories.

The symmetric cases are given in Tables 3 and 4 . As for the antisymmetric cases, the series expansion results converge quite 
Table 1

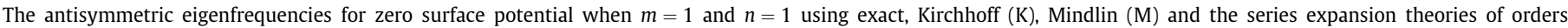
$N=3,5,7,9$.

\begin{tabular}{|c|c|c|c|c|c|c|c|c|}
\hline$a / h$ & $\Omega$ & Exact & K & $\mathrm{M}$ & $N=3$ & $N=5$ & $N=7$ & $N=9$ \\
\hline \multirow[t]{3}{*}{10} & $\Omega_{1}$ & 0.02177 & 0.02254 & 0.02176 & 0.02168 & 0.02177 & 0.02177 & 0.02177 \\
\hline & $\Omega_{2}$ & 0.6837 & - & 0.6833 & 0.6437 & 0.6862 & 0.6836 & 0.6837 \\
\hline & $\Omega_{3}$ & 0.8051 & - & 0.8063 & 0.7270 & 0.8163 & 0.8046 & 0.8051 \\
\hline \multirow[t]{3}{*}{4} & $\Omega_{1}$ & 0.1179 & 0.1412 & 0.1176 & 0.1157 & 0.1178 & 0.1179 & 0.1179 \\
\hline & $\Omega_{2}$ & 0.7436 & - & 0.7406 & 0.7038 & 0.7465 & 0.7435 & 0.7436 \\
\hline & $\Omega_{3}$ & 0.8962 & - & 0.9045 & 0.8213 & 0.9092 & 0.8956 & 0.8962 \\
\hline \multirow[t]{3}{*}{1} & $\Omega_{1}$ & 0.8609 & 2.3325 & 0.8501 & 0.7904 & 0.8530 & 0.8603 & 0.8609 \\
\hline & $\Omega_{2}$ & 1.262 & - & 1.253 & 1.253 & 1.266 & 1.262 & 1.262 \\
\hline & $\Omega_{3}$ & 1.786 & - & 2.004 & 1.610 & 1.854 & 1.774 & 1.787 \\
\hline
\end{tabular}

Table 2

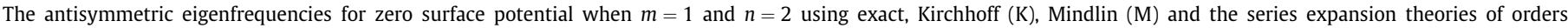
$N=3,5,7,9$.

\begin{tabular}{|c|c|c|c|c|c|c|c|c|}
\hline$a / h$ & $\Omega$ & Exact & $\mathrm{K}$ & $\mathrm{M}$ & $N=3$ & $N=5$ & $N=7$ & $N=9$ \\
\hline \multirow[t]{3}{*}{10} & $\Omega_{1}$ & 0.05470 & 0.05929 & 0.05454 & 0.05417 & 0.05469 & 0.05470 & 0.05470 \\
\hline & $\Omega_{2}$ & 0.6925 & - & 0.6905 & 0.6545 & 0.6949 & 0.6924 & 0.6925 \\
\hline & $\Omega_{3}$ & 0.8448 & - & 0.8492 & 0.7663 & 0.8572 & 0.8443 & 0.8448 \\
\hline \multirow[t]{3}{*}{4} & $\Omega_{1}$ & 0.2617 & 0.3716 & 0.2589 & 0.2528 & 0.2615 & 0.2617 & 0.2617 \\
\hline & $\Omega_{2}$ & 0.7912 & - & 0.7813 & 0.7661 & 0.7929 & 0.7912 & 0.7912 \\
\hline & $\Omega_{3}$ & 1.082 & - & 1.109 & 0.9949 & 1.105 & 1.080 & 1.082 \\
\hline \multirow[t]{3}{*}{1} & $\Omega_{1}$ & 1.533 & 6.115 & 1.487 & 1.264 & 1.486 & 1.526 & 1.532 \\
\hline & $\Omega_{2}$ & 1.805 & - & 1.830 & 1.902 & 1.840 & 1.813 & 1.806 \\
\hline & $\Omega_{3}$ & 2.328 & - & 3.143 & 2.067 & 2.374 & 2.305 & 2.330 \\
\hline
\end{tabular}

Table 3

The symmetric eigenfrequencies for zero surface potential when $m=1$ and $n=1$ using exact, Mindlin (M) and the series expansion theories of orders $N=1,3,5,7,9$.

\begin{tabular}{cllllllll}
\hline$a / h$ & $\Omega$ & Exact & $\mathrm{M}$ & $N=1$ & $N=3$ & $N=5$ & $N=7$ & $N=9$ \\
\hline 10 & $\Omega_{1}$ & 0.1001 & 0.1001 & 0.1001 & 0.1001 & 0.1001 & 0.1001 & 0.1001 \\
& $\Omega_{2}$ & 0.1761 & 0.1765 & 0.1766 & 0.1761 & 0.1761 & 0.1761 & 0.1761 \\
& $\Omega_{3}$ & 1.524 & - & - & 1.218 & 1.607 & 1.506 & 1.525 \\
& & & & & & & & \\
4 & $\Omega_{1}$ & 0.2512 & 0.2512 & 0.2518 & 0.2512 & 0.2512 & 0.2512 & 0.2512 \\
& $\Omega_{2}$ & 0.4374 & 0.4334 & 0.4448 & 0.4372 & 0.4374 & 0.4374 & 0.4374 \\
& $\Omega_{3}$ & 1.463 & - & - & 1.182 & 1.725 & 1.446 & 1.463 \\
& & & & & & & & \\
1 & $\Omega_{1}$ & 1.035 & 1.029 & 1.049 & 1.038 & 1.036 & 1.035 & 1.035 \\
& $\Omega_{2}$ & 1.271 & 1.838 & 1.904 & 1.222 & 1.280 & 1.271 & 1.271 \\
& $\Omega_{3}$ & 1.903 & - & - & 1.681 & 1.959 & 1.892 & 1.903 \\
\hline
\end{tabular}

Table 4

The symmetric eigenfrequencies for zero surface potential when $m=1$ and $n=2$ using exact, Mindlin (M) and the series expansion theories of orders $N=1,3,5,7,9$.

\begin{tabular}{cllllllll}
\hline$a / h$ & $\Omega$ & Exact & $\mathrm{M}$ & $N=1$ & $N=3$ & $N=5$ & $N=7$ & $N=9$ \\
\hline 10 & $\Omega_{1}$ & 0.1523 & 0.1523 & 0.1523 & 0.1523 & 0.1523 & 0.1523 & 0.1523 \\
& $\Omega_{2}$ & 0.2923 & 0.2938 & 0.2940 & 0.2922 & 0.2923 & 0.2923 & 0.2923 \\
& $\Omega_{3}$ & 1.486 & - & - & 1.194 & 1.642 & 1.468 & 1.488 \\
& & & & & & & & \\
4 & $\Omega_{1}$ & 0.3899 & 0.3893 & 0.3956 & 0.3902 & 0.3899 & 0.3899 & 0.3899 \\
& $\Omega_{2}$ & 0.7095 & 0.7379 & 0.7405 & 0.7053 & 0.7097 & 0.7095 & 0.7095 \\
& $\Omega_{3}$ & 1.431 & - & - & 1.175 & 1.572 & 1.416 & 1.431 \\
& & & & & & & & \\
1 & $\Omega_{1}$ & 1.708 & 1.667 & 1.796 & 1.748 & 1.719 & 1.710 & 1.708 \\
& $\Omega_{2}$ & 1.715 & 3.002 & 3.083 & 1.778 & 1.740 & 1.719 & 1.715 \\
& $\Omega_{3}$ & 2.390 & - & - & 2.277 & 2.305 & 2.386 & 2.390 \\
\hline
\end{tabular}

rapidly to the exact results as the power series orders are increased. The $N=1$ results are of the same accuracy order as Mindlin's, albeit the latter are marginally better in most cases.

\subsection{Piezoelectric plate poled in the $z$ direction}

Consider next a PZT-4 plate poled in the $z$ direction. The material constants are [27] $E_{1}=E_{2}=81.3 \mathrm{GPa}, E_{3}=64.5 \mathrm{GPa}, G_{12}=30.6 \mathrm{GPa}$, $G_{13}=G_{23}=25.6 \mathrm{GPa}, \quad v_{12}=0.329, \quad v_{13}=v_{23}=0.432, \quad e_{x 5}=e_{y 4}=$ $12.72 \mathrm{C} / \mathrm{m}^{2}, \quad e_{z 1}=e_{z 2}=-5.2 \mathrm{C} / \mathrm{m}^{2}, \quad e_{z 3}=15.08 \mathrm{C} / \mathrm{m}^{2}, \quad \epsilon_{x x}=\epsilon_{y y}=$ $1475 \epsilon_{0}$, and $\epsilon_{z z}=1300 \epsilon_{0}$ where $\epsilon_{0}=8.85 \cdot 10^{-12} \mathrm{C} / \mathrm{Vm}$. Here $c_{66}=\left(c_{12}-c_{22}\right) / 2$ is only approximately fulfilled using these material constants. Adopting the general anisotropic plate equations derived in Section 4, sets of antisymmetric and symmetric plate equations are obtained in a straightforward manner. These plate equations are different to the ones poled in the $x$ direction presented in Section 6, although both poled directions involve terms of the same differential orders. Note that the plate equations poled in the $z$ direction are also asymptotically correct to the studied order $N=5$.

Consider simply supported quadratic plates, where the elastic boundary conditions are as in Section 7.1 while the potential is zero at all edges. Tables 5-8 illustrate the three lowest eigenfrequencies $\tilde{\omega}_{i}=\omega_{i} / 100$ when $m=n=1$ for both antisymmetric and symmetric motions, using three different plate thicknesses $a / h$. Adopting this frequency normalization, the plate geometry is to be stated which here is set to $2 h=0.01$. Comparisons are made to Benjeddou and Deü [48] and Robaldo et al. [20] for some cases studied therein. The theory in [48] is based on Mindlin assumptions without shear correction factors and thus should preferably be compared to the present theory of order $N=3$ in the antisymmetric case and $N=1$ in the symmetric case. The plate theory in [20] considers a series expansion theory of exponential order 4 solved using FEM, and thus may well be compared to the present theory for $N=4$. The presented lists of eigenfrequencies involve quite a few significant digits considering the approximate nature of the given material and physical constants. The reason for this 
Table 5

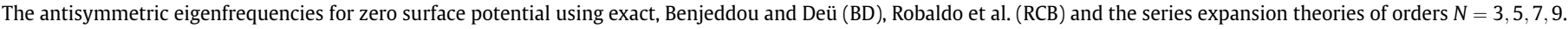

\begin{tabular}{|c|c|c|c|c|c|c|c|c|}
\hline$a / h$ & $\tilde{\omega}$ & Exact & $\mathrm{BD}$ & $\mathrm{RCB}$ & $N=3$ & $N=5$ & $N=7$ & $N=9$ \\
\hline \multirow[t]{3}{*}{50} & $\tilde{\omega}_{1}$ & 746.752 & \multirow[t]{3}{*}{746.837} & \multirow[t]{3}{*}{746.834} & 746.581 & 746.730 & 746.752 & 746.752 \\
\hline & $\tilde{\omega}_{2}$ & 502,895 & & & 452,815 & 509,821 & 502,610 & 502,903 \\
\hline & $\tilde{\omega}_{3}$ & 503,469 & & & 453,359 & 510,418 & 503,182 & 503,477 \\
\hline \multirow[t]{3}{*}{10} & $\tilde{\omega}_{1}$ & 18013.4 & \multirow[t]{3}{*}{18071.1} & & 17919.6 & 18012.6 & 18013.4 & 18013.4 \\
\hline & $\tilde{\omega}_{2}$ & 508,625 & & & 459,171 & 515,474 & 508,343 & 508,633 \\
\hline & $\tilde{\omega}_{3}$ & 522,320 & & & 472,052 & 529,764 & 522,000 & 522,329 \\
\hline \multirow[t]{3}{*}{4} & $\tilde{\omega}_{1}$ & 96929.9 & \multirow[t]{3}{*}{98246.6} & \multirow[t]{3}{*}{97321.4} & 94718.4 & 96892.0 & 96929.6 & 96929.9 \\
\hline & $\tilde{\omega}_{2}$ & 538,885 & & & 492,479 & 545,354 & 538,619 & 538,892 \\
\hline & $\tilde{\omega}_{3}$ & 609,186 & & & 556,144 & 619,754 & 608,653 & 609,205 \\
\hline
\end{tabular}

Table 6

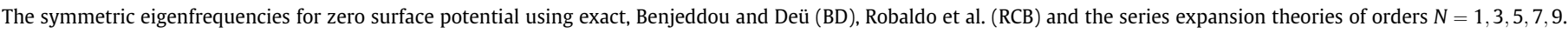

\begin{tabular}{|c|c|c|c|c|c|c|c|c|c|}
\hline$a / h$ & $\tilde{\omega}$ & Exact & $\mathrm{BD}$ & $\mathrm{RCB}$ & $N=1$ & $N=3$ & $N=5$ & $N=7$ & $N=9$ \\
\hline \multirow[t]{3}{*}{50} & $\tilde{\omega}_{1}$ & 15540.4 & 15540.4 & 15542.0 & 15540.4 & 15540.4 & 15540.4 & 15540.4 & 15540.4 \\
\hline & $\tilde{\omega}_{2}$ & 26828.0 & 26831.7 & 26828.2 & 26832.6 & 26828.0 & 26828.0 & 26828.0 & 26828.0 \\
\hline & $\tilde{\omega}_{3}$ & $1,004,344$ & & & - & 783,502 & $1,094,561$ & 984,390 & $1,006,587$ \\
\hline \multirow[t]{3}{*}{10} & $\tilde{\omega}_{1}$ & 77702.1 & 77702.1 & & 77702.1 & 77702.1 & 77702.1 & 77702.1 & 77702.1 \\
\hline & $\tilde{\omega}_{2}$ & 133,695 & 134,158 & & 134,273 & 133,691 & 133,695 & 133,695 & 133,695 \\
\hline & $\tilde{\omega}_{3}$ & 988,021 & & & - & 776,834 & $1,114,956$ & 970,449 & 989,934 \\
\hline \multirow[t]{3}{*}{4} & $\tilde{\omega}_{1}$ & 194,255 & 194,255 & 194,264 & 194,255 & 194,255 & 194,255 & 194,255 & 194,255 \\
\hline & $\tilde{\omega}_{2}$ & 327,663 & 335,396 & 327,670 & 337,105 & 327,245 & 327,674 & 327,663 & 327,663 \\
\hline & $\tilde{\omega}_{3}$ & 958,922 & & & - & 766,465 & $1,018,717$ & 944,340 & 960,404 \\
\hline
\end{tabular}

Table 7

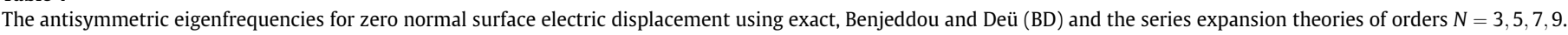

\begin{tabular}{|c|c|c|c|c|c|c|c|}
\hline$a / h$ & $\tilde{\omega}$ & Exact & $\mathrm{BD}$ & $N=3$ & $N=5$ & $N=7$ & $N=9$ \\
\hline \multirow[t]{3}{*}{50} & $\tilde{\omega}_{1}$ & 746.873 & 746.957 & 746.702 & 746.873 & 746.873 & 746.873 \\
\hline & $\tilde{\omega}_{2}$ & 502,895 & & 452,815 & 509,821 & 502,610 & 502,903 \\
\hline & $\tilde{\omega}_{3}$ & 586,240 & & 512,371 & 603,332 & 585,385 & 586,227 \\
\hline \multirow[t]{3}{*}{10} & $\tilde{\omega}_{1}$ & 18077.8 & 18136.1 & 17982.9 & 18077.5 & 18077.8 & 18077.8 \\
\hline & $\tilde{\omega}_{2}$ & 508,625 & & 459,171 & 515,474 & 508,343 & 508,633 \\
\hline & $\tilde{\omega}_{3}$ & 604,752 & & 530,530 & 623,173 & 603,805 & 604,736 \\
\hline \multirow[t]{3}{*}{4} & $\tilde{\omega}_{1}$ & 98231.7 & 99634.9 & 95938.1 & 98195.5 & 98231.4 & 98231.7 \\
\hline & $\tilde{\omega}_{2}$ & 538,885 & & 492,479 & 545,354 & 538,619 & 538,892 \\
\hline & $\tilde{\omega}_{3}$ & 690,767 & & 612,873 & 717,862 & 689,262 & 690,735 \\
\hline
\end{tabular}

Table 8

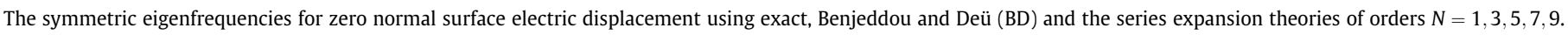

\begin{tabular}{|c|c|c|c|c|c|c|c|c|}
\hline$a / h$ & $\tilde{\omega}$ & Exact & $\mathrm{BD}$ & $N=1$ & $N=3$ & $N=5$ & $N=7$ & $N=9$ \\
\hline \multirow[t]{3}{*}{50} & $\tilde{\omega}_{1}$ & 15540.4 & 15540.4 & 15540.4 & 15540.4 & 15540.4 & 15540.4 & 15540.4 \\
\hline & $\tilde{\omega}_{2}$ & 29153.3 & 29155.6 & 29157.8 & 29153.3 & 29153.3 & 29153.3 & 29153.3 \\
\hline & $\tilde{\omega}_{3}$ & $1,004,612$ & & - & 783,507 & $1,035,593$ & 984,552 & $1,006,872$ \\
\hline \multirow[t]{3}{*}{10} & $\tilde{\omega}_{1}$ & 77702.1 & 77702.1 & 77702.1 & 77702.1 & 77702.1 & 77702.1 & 77702.1 \\
\hline & $\tilde{\omega}_{2}$ & 145,221 & 145,610 & 145,789 & 145,215 & 145,221 & 145,221 & 145,221 \\
\hline & $\tilde{\omega}_{3}$ & 990,953 & & - & 776,867 & $1,030,751$ & 972,401 & 993,008 \\
\hline \multirow[t]{3}{*}{4} & $\tilde{\omega}_{1}$ & 194,255 & 194,255 & 194,255 & 194,255 & 194,255 & 194,255 & 194,255 \\
\hline & $\tilde{\omega}_{2}$ & 355,110 & 361,926 & 364,473 & 354,338 & 355,133 & 355,109 & 355,110 \\
\hline & $\tilde{\omega}_{3}$ & 960,103 & & - & 766,832 & $1,013,234$ & 945,063 & 961,646 \\
\hline
\end{tabular}

is to make comparisons more transparent since such lists are presented in $[20,48]$.

Tables 5 and 6 illustrate the three lowest eigenfrequencies for short-circuited antisymmetric and symmetric motions, respectively. The accuracies using the present series method are of the same order as for the plates poled in the $x$ direction presented in Section 7.1. The series results converge to the exact results in all cases studied, where a few more terms are needed for the higher frequencies (up to $N=13$ ). As for the other theories, the Benjeddou and Deü theory is slightly more accurate for the eigenfrequencies than the present theory for $N=3$ (antisymmetric) and $N=1$ (symmetric), respectively. By increasing the series order one step to $N=5$ (antisymmetric) and $N=3$ (symmetric), the present results are improved considerably. As for the results due to 
Table 9

Eigenfrequencies for laminated plate with zero normal surface potential using exact, Benjeddou and Deü (BD) and the series expansion theories of orders $N=3,5,7,9$.

\begin{tabular}{|c|c|c|c|c|c|c|c|}
\hline$a / h$ & $\tilde{\omega}$ & Exact & $\mathrm{BD}$ & $N=3$ & $N=5$ & $N=7$ & $N=9$ \\
\hline \multirow[t]{4}{*}{50} & $\tilde{\omega}_{1}$ & 633.417 & 633.666 & 633.192 & 633.393 & 633.415 & 633.417 \\
\hline & $\tilde{\omega}_{2}$ & 16431.1 & 16432.5 & 16430.7 & 16431.1 & 16431.1 & 16431.1 \\
\hline & $\tilde{\omega}_{3}$ & 28535.2 & 28537.6 & 28534.0 & 28535.2 & 28535.2 & 28535.2 \\
\hline & $\tilde{\omega}_{4}$ & 268,118 & 292,032 & 238,030 & 271,728 & 267,900 & 268,138 \\
\hline \multirow[t]{4}{*}{4} & $\tilde{\omega}_{1}$ & 72174.4 & 73969.5 & 70474.3 & 72122.2 & 72171.9 & 72174.3 \\
\hline & $\tilde{\omega}_{2}$ & 194,760 & 197,165 & 193,744 & 194,770 & 194,760 & 194,760 \\
\hline & $\tilde{\omega}_{3}$ & 306,209 & 329,399 & 302,768 & 306,159 & 306,205 & 306,209 \\
\hline & $\tilde{\omega}_{4}$ & 337,107 & 345,027 & 301,741 & 340,906 & 336,821 & 337,134 \\
\hline
\end{tabular}

Table 10

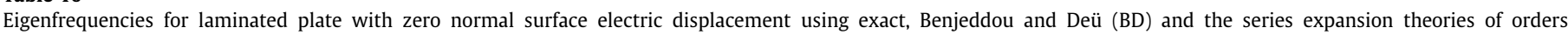
$N=3,5,7,9$.

\begin{tabular}{|c|c|c|c|c|c|c|c|}
\hline$a / h$ & $\tilde{\omega}$ & Exact & $\mathrm{BD}$ & $N=3$ & $N=5$ & $N=7$ & $N=9$ \\
\hline \multirow[t]{4}{*}{50} & $\tilde{\omega}_{1}$ & 633.487 & 633.735 & 633.280 & 633.469 & 633.486 & 633.487 \\
\hline & $\tilde{\omega}_{2}$ & 16440.9 & 16442.2 & 16440.6 & 16440.9 & 16440.9 & 16440.9 \\
\hline & $\tilde{\omega}_{3}$ & 28555.3 & 28557.7 & 28554.2 & 28555.3 & 28555.3 & 28555.3 \\
\hline & $\tilde{\omega}_{4}$ & 271,222 & 295,866 & 240,684 & 274,472 & 271,007 & 271,245 \\
\hline \multirow[t]{4}{*}{4} & $\tilde{\omega}_{1}$ & 72191.5 & 74,006 & 70483.8 & 72141.7 & 72187.8 & 72191.3 \\
\hline & $\tilde{\omega}_{2}$ & 194,881 & 197,280 & 194,072 & 194,889 & 194,881 & 194,881 \\
\hline & $\tilde{\omega}_{3}$ & 306,539 & 329,862 & 302,839 & 306,501 & 306,534 & 306,539 \\
\hline & $\tilde{\omega}_{4}$ & 337,196 & 345,226 & 301,923 & 340,508 & 336,930 & 337,223 \\
\hline
\end{tabular}

Robaldo et al., these show accuracies similar to $N=3$ for thin plates and closer to $N=5$ for thick plates. Eigenfrequencies for open-circuited plates are presented in Tables 7 and 8 . The results show a similar pattern as for the short-circuited case.

It should be noted that the calculated exact eigenfrequencies presented in Tables 5-8 are identical to the results due to Heyliger and Saravanos [27].

\subsection{Piezoelectric laminate}

In order to further illustrate the present expansion method, eigenfrequencies for laminated piezoelectric plates are calculated. There are much work on various laminated plate structures with piezoelectric layers bonded to the plate faces [19-21,24,27,32, $33,46,48]$. Among these, Heyliger and Saravanos [27] present exact $3 \mathrm{D}$ results for three and five layered simply supported plates. Here three-layer piezoelectric plates are considered, where comparisons are made to the exact theory [27] and the approximate Mindlin theory due to Benjeddou and Deü [48]. Consider a plate with total thickness $2 h$ that consists of a PZT- 4 core of thickness $h$ (material constants as in Section 7.2) surrounded symmetrically by orthotropic PVDF layers with material constants [27] $E_{1}=237,0 \mathrm{GPa}$, $E_{2}=23.2 \mathrm{GPa}, E_{3}=10.5 \mathrm{GPa}, G_{12}=6.43 \mathrm{GPa}, G_{13}=4.4 \mathrm{GPa}, G_{23}=$ $2.15 \mathrm{GPa}, \quad v_{12}=0.154, \quad v_{13}=0.178, v_{23}=0.177, \quad e_{x 5}=e_{y 4}=$ $-0.01 \mathrm{C} / \mathrm{m}^{2}, \quad e_{z 1}=-0.13 \mathrm{C} / \mathrm{m}^{2}, \quad e_{z 2}=-0.14 \mathrm{C} / \mathrm{m}^{2}, \quad e_{z 3}=$ $-0.28 \mathrm{C} / \mathrm{m}^{2}, \epsilon_{x x}=12.5 \epsilon_{0}, \epsilon_{y y}=11.98 \epsilon_{0}$, and $\epsilon_{z z}=11.98 \epsilon_{0}$ where $\epsilon_{0}=8.85 \cdot 10^{-12} \mathrm{C} / \mathrm{Vm}$.

The derivation process for the present series expansion techniques follows from Mauritsson et al. [42] applied to plates with piezoelectric core material. By splitting the solution process in symmetric and antisymmetric parts, eigenfrequencies are obtained for different truncation orders. The procedure is more involved than for a single plate layer, as separate series expansion systems are introduced for the layers. The coupling conditions at the layer interfaces are modeled at each point, and the free surface conditions are fulfilled at $z= \pm h$ just as for the single plate layer.

The four lowest eigenfrequencies $\tilde{\omega}_{i}=\omega_{i} / 100$ when $m=n=1$ and $2 h=0.01$ are presented in Tables 9 and 10 for short-circuited and open-circuited laminated plates, respectively. Both cases show similar results regarding eigenfrequencies and accuracies for the different approximative theories. In each case, the first and fourth eigenfrequencies correspond to antisymmetric motion while the middle frequencies are for symmetric motion. The series expansion approximations show similar behavior as for the single layer cases in Tables $5-8$, albeit the rates of convergence are slightly lower in the laminated cases. This effect is most notable for the first symmetric mode $\tilde{\omega}_{2}$ for the thicker plate. Hence, the results indicate that the present method converges to the exact results for all the cases studied, although higher order truncations $N>9$ have not been studied. Similar to the single plate layer, the results due to the Benjeddou and Deü theory are slightly more accurate for antisymmetric motion when compared to the present theory of $\operatorname{order} N=3$. The opposite holds for symmetric motions.

\section{Concluding remarks}

Plate equations and corresponding edge boundary conditions for a fully anisotropic piezoelectric rectangular plate are derived to arbitrary order using a systematic power series expansion approach. The plate equations are given on a compact form using recursively defined matrix differential operators, where the set of equations are variationally consistent and asymptotically correct to all studied orders. These equations are simplified for an orthotropic $2 \mathrm{~mm}$ material poled in an in-plane direction, where the plate equations and edge boundary conditions are given explicitly. Numerical comparisons for dispersion curves and the cross sectional fields (potential, displacements, stresses) are made between the asymptotic plate theory, the Kirchhoff theory, the Mindlin theory and exact theory. Eigenfrequencies are presented for simply supported plates poled either in the plate plane or normal to the plate plane. Both single and laminated plates are studied for different plate thicknesses using various plate theories. The results illustrate the accuracies using different series truncations for the various eigenmodes where the rate of convergence is high in most cases, including the more complicated laminated plate structures. 
The derived system of plate equations may either be used to low truncation order as engineering plate equations, or to higher order acting as three dimensional benchmark results. A possible application for the former case is to develop piezoelectric plate element for finite element codes. Hereby one benefits from the accurate results using one of the present lower order theories, and at the same time the number of elements can be heavily reduced compared to using three dimensional elements. As for the latter case using higher order truncations, this calls for an alternative way of obtaining solutions to the three dimensional equations [49]. This is especially of interest for more complicated anisotropic configurations.

Future work is to develop higher order theories for more complicated material configurations, such as involving functionally graded, porous or micro materials. For such configurations, several different plate theories have appeared, and the present systematic approach would render equations that are variationally consistent and directly based on the three dimensional theories. Related work based on power series expansion and recursion relations have previously been carried out on isotropic materials such as porous [50] and functionally graded [49] plates, and is currently directed towards micro plates. Another area of interest is to further study laminates involving multiple elastic plates embedded in piezoelectric layers [33]. This may be accomplished by adopting the present piezoelectric plate equations and the corresponding equations for anisotropic plates [35], using generalizations of the methodology presented in $[42,43]$.

\section{Acknowledgements}

The present work is sponsored by the Swedish Research Council (VR) and this is gratefully acknowledged. The authors would like to thank Prof. Anders Boström for interesting discussions during the process of this work.

\section{References}

[1] Wang J, Yang J. Higher-order theories of piezoelectric plates and applications. Appl Mech Rev 2000;53:87-99.

[2] Gopinathan SV, Varadan VV, Varadan VK. A review and critique of theories for piezoelectric laminates. Smart Mater Struct 2000;9:24-48.

[3] Bisegna P, Caruso G. Evaluation of higher-order theories of piezoelectric plates in bending and in stretching. Int J Solids Struct 2001;38:8805-30.

[4] Wu CP, Chiu K-H, Wang Y-M. A review on the three-dimensional analytical approaches of multilayered and functionally graded piezoelectric plates and shells. CMC 2008;8:93-132.

[5] Mindlin RD. Forced thickness-shear and flexural vibrations of piezoelectric crystal plates. J Appl Phys 1952;23:83-8.

[6] Tiersten HF, Mindlin RD. Forced vibrations of piezoelectric crystal plates. Quart Appl Math 1962;20:107-19.

[7] Mindlin RD. High frequency vibrations of piezoelectric crystal plates. Int J Solids Struct 1972;8:895-906.

[8] Bugdayci N, Bogy DB. A two-dimensional theory for piezoelectric layers used in electro-mechanical transducers-I Derivation. Int J Solids Struct 1981;17:1159-78.

[9] Lee PCY, Syngellakis S, Hou JP. A two-dimensional theory for high-frequency vibrations of piezoelectric crystal plates with or without electrodes. J App Phys 1987:61:1249-62.

[10] Lee PCY, Yu JD, Lin WS. A new two-dimensional theory for vibrations of piezoelectric crystal plates with electroded faces. J Appl Phys 1998;83:1213-23.

[11] Gao J-X, Shen Y-P, Wang J. Three dimensional analysis for free vibration of rectangular composite laminates with piezoelectric layers. J Sound Vib 1998;213:383-90.

[12] Fernandes A, Pouget J. Accurate modelling of piezoelectric plates: singlelayered plate. Arch Appl Mech 2001;71:509-24.

[13] Tiersten HF. A derivation of two-dimensional equations for the vibration of electroded piezoelectric plates using an unrestricted thickness expansion of the electric potential. In: IEEE Int Freq Contr Symp PDA Exhibition; 2001. p. 571-79.

[14] Tiersten HF. On the thickness expansion of the electric potential in the determination of two-dimensional equations for the vibration of electroded piezoelectric plates. J Appl Phys 2002;91:2277-83.
[15] Batra RC, Vidoli S. Higher-order piezoelectric plate theory derived from a three-dimensional variational principle. AIAA J 2002;40:91-104.

[16] Vel SS, Mewer RC, Batra RC. Analytical solution for the cylindrical bending vibration of piezoelectric composite plates. Int J Solids Struct 2004;41:1625-43.

[17] Yang J, Zhou H, Wang Z. Vibrations on an asymmetrically electroded piezoelectric plate. IEEE Trans Ultrason, Ferroelect, Freq Contr 2005; $52: 2031-8$

[18] Baillargeon BP, Vel SS. Exact solution for the vibration and active damping of composite plates with piezoelectric shear actuators. I Sound Vib 2005; 282:781-804.

[19] Kapuria S, Achary GGS. A coupled zigzag theory for the dynamics of piezoelectric hybrid cross-ply plates. Arch Appl Mech 2005;75:42-57.

[20] Robaldo A, Carrera E, Benjeddou A. A unified formulation for finite element analysis of piezoelectric adaptive plates. Comput Struct 2006;84:1494-505.

[21] Kant T, Shieyekar SM. Cylindrical bending of piezoelectric laminates with a higher order shear and normal deformation theory. Comput Struct 2008; 86:1594-603.

[22] Vetyukov Y, Kuzin A, Krommer M. Asymptotic splitting in the threedimensional problem of elasticity for non-homogeneous piezoelectric plates. Int J Solids Struct 2011;48:12-23.

[23] Madureira AL. Hierarchical modeling of piezoelectric plates. Appl Math Model 2012;36:3555-69.

[24] Askari Farsangi MA, Saidi AR, Batra RC. Analytical solution for free vibrations of moderately thick hybrid piezoelectric laminated plates. J Sound Vib 2013;332:5981-98.

[25] Tiersten HF. Linear piezoelectric plate vibrations. New York: Plenum; 1969.

[26] Ray MC, Rao KM, Samanta B. Exact analysis of coupled electroelastic behaviour of a piezoelectric plate under cylindrical bending. Comput Struct 1992; 45:667-77.

[27] Heyliger P, Saravanos DA. Exact free-vibration analysis of laminated plates with embedded piezoelectric layers. J Acoust Soc Am 1995;98:1547-57.

[28] Bisegna P, Maceri F. An exact three-dimensional solution for simply supported rectangular piezoelectric plates. J Appl Mech 1996;63:628-38.

[29] Wang J, Yu J, Yong Y, Imai T. A new theory for electroded piezoelectric plates and its finite element application for the forced vibration of quartz crystal resonators. Int J Solids Struct 2000;37:5653-73.

[30] Kögl M, Bucalem ML. A family of piezoelectric MITC plate elements. Comput Struct 2005;83:1277-97.

[31] Braess D, Kaltenbacher M. Efficient 3D-finite element formulation for thin mechanical and piezoelectric structures. Int J Numer Methods Eng 2008; 73:147-61.

[32] Carrera E, Brischetto S, Cinefra M. Variable kinematics and advanced variational statements for free analysis of piezoelectric plates and shells. CMES 2010:65:259-341.

[33] Carrera E, Brischetto S, Nali P. Plates and shells for smart structures. Wiley; 2011.

[34] Man H, Song C, Gao W, Tin-Loi F. Semi-analytical analysis for piezoelectric plate using the scaled boundary finite-element method. Comput Struct 2013;137:47-62.

[35] Mauritsson K, Folkow PD, Boström A. Dynamic equations for a fully anisotropic elastic plate. J Sound Vib 2011;330:2640-54.

[36] Boström A. On wave equations for elastic rods. Z Angew Math Mech 2000; 80:245-51.

[37] Folkow PD, Mauritsson K. Dynamic higher-order equations for finite rods. Q J Mech Appl Math 2010;63:1-22.

[38] Abadikhah H, Folkow PD. A hierarchy of dynamic equations for solid isotropic circular cylinders. Wave Motion 2013;51:206-21.

[39] Hägglund AM, Folkow PD. Dynamic cylindrical shell equations by power series expansions. Int J Solids Struct 2008;45:4509-22.

[40] Boström A, Johansson G, Olsson P. On the rational derivation of a hierarchy of dynamic equations for a homogeneous, isotropic, elastic plate. Int J Solids Struct 2001;38:2487-501.

[41] Johansson G, Niklasson AJ. Approximate dynamic boundary conditions for a thin piezoelectric layer. Int J Solids Struct 2003;40:3477-92.

[42] Mauritsson K, Boström A, Folkow PD. Modelling of thin piezoelectric layers on plates. Wave Motion 2008;45:616-28.

[43] Mauritsson K. Modelling of finite piezoelectric patches: comparing an approximate power series expansion theory with exact theory. Int J Solids Struct 2009;46:1053-65.

[44] Losin NA. On the equivalence of dispersion relations resulting from RayleighLamb frequency equation and the operator plate model. J Vibr Acoust 2001; 123:417-20.

[45] Auld BA. Acoustic fields and waves in solids. Malabar, FL: Krieger; 1990.

[46] Ballhause D, D'Ottavio M, Kröplin B, Carrera E. A unified formulation to assess multilayered theories for piezoelectric plates. Comput Struct 2005;83:1217-35.

[47] Reddy JN. Mechanics of laminated composite plates: theory and analysis. Boca Raton: CRC Press; 1997.

[48] Benjeddou A, Deü J-F. A two-dimensional closed-form solution for the freevibrations analysis of piezoelectric sandwich plates. Int J Solids Struct 2002; 39:1463-86.

[49] Vel SS, Batra RC. Three-dimensional exact solution for the vibration of functionally graded rectangular plates. J Sound Vib 2004;272:703-30.

[50] Folkow PD, Johansson M. Dynamic equations for fluid-loaded porous plates using approximate boundary conditions. J Acoust Soc Am 2009;125:2954-66. 